\title{
Transatlantica
}

Revue d'études américaines. American Studies Journal

$1 \mid 2012$

Le roman policier, littérature transatlantique / Maisons

Hantées

\section{Le Krimi sous le Troisième Reich : une invention de l'étranger}

\section{Vincent Platini}

\section{CpenEdition}

\section{Journals}

Édition électronique

URL : https://journals.openedition.org/transatlantica/5729

DOI : $10.4000 /$ transatlantica.5729

ISSN : 1765-2766

Éditeur

Association française d'Etudes Américaines (AFEA)

Référence électronique

Vincent Platini, "Le Krimi sous le Troisième Reich : une invention de l'étranger », Transatlantica [En ligne], 1 | 2012, mis en ligne le 03 janvier 2013, consulté le 01 février 2023. URL : http://

journals.openedition.org/transatlantica/5729; DOI : https://doi.org/10.4000/transatlantica.5729

Ce document a été généré automatiquement le 1 février 2023.

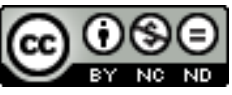

Creative Commons - Attribution - Pas d'Utilisation Commerciale - Pas de Modification 4.0 International - CC BY-NC-ND 4.0

https://creativecommons.org/licenses/by-nc-nd/4.0/ 


\title{
Le Krimi sous le Troisième Reich : une invention de l'étranger
}

\author{
Vincent Platini
}

\section{Introduction : dévalorisation du genre et marges de liberté}

1 L'étude du roman policier (Kriminalroman ou Krimi) sous le Troisième Reich touche moins aux énigmes policières qu'à une éclipse littéraire. Bien que le pays puisse se targuer de quelques illustres précurseurs en matière de littérature criminelle ${ }^{1}$, la critique et les institutions ont souvent considéré que le polar allemand avait émergé vers 1960 (avec Hansjörg Martin, Richard Hey, Gert Prokop, Horst Bosetzky et Michael Molsner) après avoir longtemps été le domaine réservé de la littérature étrangère. Ainsi, la seule bibliothèque publique spécialisée-la Krimibibliothek de Brême-possède un fonds composé de romans publiés exclusivement après 1965. Assez récemment encore, certains critiques niaient l'existence du genre policier sous le régime nazi (Alf Mayer-Ebeling, 1997, 205). Cette ignorance s'explique par l'accès difficile au matériau de recherche et, pendant longtemps, une absence de repères bibliographiques. Quand les collections n'appartiennent pas à des particuliers, les catalogues des bibliothèques sont lacunaires. Enfin, les rares études consacrées au Krimi sous le Troisième Reich l'ont souvent réduit à un simple outil de propagande ${ }^{2}$ qui ne relève pas des études littéraires à proprement parler ${ }^{3}$.

Les travaux publiés depuis une dizaine d'années permettent de nuancer le propos. L'imposante bibliographie de Mirko Schädel (2006) démontre que le roman criminel a perduré sous le nazisme tandis que les études de Kate Sturge et Markus Spieker ont souligné l'engouement du public allemand pour la production anglo-américaine. En outre, les recherches que mène Carsten Würmann depuis une dizaine d'années sont un cadre précieux pour l'étude de la production et des motifs récurrents sous le Troisième Reich. Plus largement, les ouvrages de Jan-Pieter Barbian et, récemment, de Christian Adam soulignent la diversité des pratiques quant à l'édition et la réception des œuvres. 
À travers les luttes d'influence des différents offices de contrôle, ou encore les marges de manœuvre laissées aux acteurs du marché du livre, la littérature-notamment policière-apparaît de moins en moins monolithique. L'inféodation du Krimi au Troisième Reich est loin d'être évidente.

3 La liberté-très relative-accordée au roman policier peut s'expliquer en termes économiques, idéologiques et structurels. Sous le national-socialisme, la production culturelle fonctionne encore dans une économie de marché. Le souci de gagner de l'argent, donc de plaire au public, a tempéré la rigueur de l'idéologie. À en croire le discours officiel, la censure n'a même pas lieu d'être sous le Troisième Reich. Un article du Börsenblatt ${ }^{4} y$ voit un moyen douteux («fragwürdige[s] Mittel ») et explique : « La promotion nationale-socialiste de la littérature doit se faire de manière positive. La seule méthode assurée de succès à long terme, est de vaincre et de supplanter les mauvais livres par les bons $»^{5}$ (Hoyer, 303). En effet, des prix et des labelsaccompagnés de dotations ou d'exonérations d'impôt-récompensent les œuvres fidèles au régime. Les considérations politiques et commerciales ne s'excluent pas; elles se concurrencent. Même les maisons d'édition directement contrôlées par la Deutsche Arbeitsfrontétaient susceptibles de publier des livres s'écartant de la ligne du Parti : les professions de foi de Robert Ley, directeur de la DAF, pouvaient couvrir des intérêts commerciaux (Adam, 27). Cette perspective économique explique la survie du Krimi : quand bien même le genre déplaisait au régime, les éditeurs ne s'en sont pas détournés car il continuait d'être rentable ${ }^{7}$. L'enjeu commercial prévaut tant que l'on n'enfreint aucun impératif idéologique majeur.

4 Or, cette production est longtemps restée en marge de la sphère politique. Dans le domaine artistique, la "mise au pas " (Gleichschaltung) concerne d'abord la "grande " littérature et le cinéma. Si le 7e art - outil d'une propagande de masse - fait l'objet de soins particuliers, il serait inconvenant que la paralittérature-qualifiée de Schundliteratur (« littérature malsaine »)-fasse campagne pour le Troisième Reich. Son kitsch souillerait le national-socialisme et sa composante commerciale l'exposerait à l'accusation d'opportunisme politique. Cette discrimination vaut surtout pour le début du régime. La loi de mai 1933 sur la protection des symboles nationaux (Gesetz zum Schutze der nationalen Symbole) empêche la récupération de l'apparat nazi par la culture de masse (Dyke, 251). Trop dévalorisé pour faire œuvre de propagande, le Krimi garde des apparences apolitiques.

5 Enfin, régime totalitaire ne signifie pas omnipotence. Bien que les évaluations chiffrées soient sujettes à caution, la production de Krimis reste assez massive pour déborder les instances de censure - surtout durant leurs premières années de mise en place. Même les organisations les plus enragées comme l'«Amt Rosenberg $»^{8}$ ne parviennent à examiner que la moitié des livres paraissant chaque année (Sturge, 98). Par ailleurs, si les écrivains et les traducteurs de métier, les éditeurs, les bibliothécaires et les libraires à plein-temps étaient obligés d'adhérer à la Chambre du livre du Reich (Reichsschrifttumskammer, RSK, fondée le 22 septembre 1933), les dilettantes n'y étaient pas contraints. Or, de nombreuxromans policiers sont le fait d'amateurs, tel Josef Hermann (pseudonyme de Bernd Carstens) dispensé d'inscription selon le registre de la RSK en $1942^{9}$ et auteur d'un Krimi pour le moins ambigu. Certes, leurs écrits restaient contrôlés puisqu'ils devaient être soumis à la Chambre pour qu'on leur délivre paradoxalement une dispense (Befreiung) d'adhésion. Toutefois, la dépendance financière de ces auteurs à l'écriture, la crainte d'être exclus de la RSK, donc la pression 
idéologique, étaient bien moindres que chez les écrivains de métier ${ }^{10}$. De même, beaucoup de bibliothèques de prêts privées n'étaient qu'une activité complémentaire pour les commerçants ou les femmes travaillant à domicile (Sturge, 77 ; Stieg, 171). En marge de la profession, ces acteurs négligés ont joué un rôle important dans la circulation du Krimi. Malgré tous ses efforts, le régime n'a jamais pu complètement assujettir cette littérature méprisée. Ainsi, en des termes qui prêtent à sourire, un article du Börsenblatt énumère les instances de contrôle culturel pour déplorer que subsistent des écueils, avant de conclure : "Ce qu'il [l'État] ne peut pas faire : écrire luimême la littérature populaire. Voilà en quoi réside la principale difficulté ${ }^{11}$ (Hasper, 608 , souligné dans le texte).

6 Ces quelques remarques sont propédeutiques à l'étude du Krimi et de sa relation à l'aire anglo-américaine. On peut considérer le roman policier du Troisième Reich comme un produit de consommation culturelle, importé de l'étranger et échappant partiellement à l'emprise idéologique du régime. Est-ce à dire que le genre a pu représenter un espace de circulation interculturelle dans une aire particulièrement hostile aux apports extérieurs ? Ou s'est-il adapté à la xénophobie ambiante au point de rejeter sa matrice étrangère? La domestication du roman policier par l'idéologie nazie parait difficile alors que les transferts culturels - et notamment la traduction littéraire (Sturge, 82-125) - sont dévalorisés dans les discours en vigueur: les œuvres étrangères comportent une altérité irréductible puisque chaque culture est spécifique à la race dont elle émane. On ne s'étonnera donc pas que le roman policier ait pu être considéré comme un corps étranger - donc nuisible - à l'Allemagne. Cependant, une possible acclimatation du genre à l'aire nazie a aussi été évoquée, notamment par ceux (auteurs, éditeurs) qui tentaient de concilier leurs intérêts avec la nouvelle donne idéologique. L'accusation d'Undeutschtum souvent lancée contre le roman policier laisse apparaître la possibilité d'un Krimi national, conforme au Deutschtum, à l'esprit germanique. Le roman étranger a-t-il alors servi de modèle ou de repoussoir? La question est bien entendu à nuancer: cette dichotomie ne saurait être parfaitement hermétique et, surtout, il faudrait déterminer quelle aire étrangère est visée. L'image du genre et de la culture allogène qu'il incarne varie selon la disponibilité des œuvres sur le marché disponibilité elle-même très fluctuante selon les périodes et les publics. En outre, la production policière est déjà empreinte d'un antagonisme entre les romans d'inspiration anglaise et américaine : un Krimi national, s'il se constitue à rebours d'une tradition littéraire, pourrait ironiquement se rapprocher d'un autre courant étranger.

7 Ces considérations soulèvent la question de l'ambivalence idéologique du roman policier, toujours suspecté de servir la société de contrôle. Peter Nusser $(1992,32)$ souligne que le genre fait du lecteur un collaborateur de la loi et de l'ordre, soulagé de voir la paix sociale restaurée. Que le roman policier ait survécu aux régimes totalitaires prouve qu'il n'est pas forcément le produit d'une culture démocratique mais plutôt la confirmation du système disciplinaire (ibid., 162) ${ }^{12}$. De fait, cette logique de contrôle, théorisée par Foucault, et ses corollaires (rationalisation et médicalisation de la justice, invention de la criminologie et de la délinquance, modernisation de la police, etc.) se développent au cours du xixe siècle en même temps que le genre policier. Produit et acteur de cette évolution, le roman criminel est un révélateur des logiques de pouvoir : le Krimi a-t-il évolué en fonction des changements de l'appareil policier du Troisième Reich? S'agit-il d'un véritable saut qualitatif ou d'une continuité, d'un durcissement des romans qui paraissent dans les pays démocratiques ? En effet, Foucault $(1975,258)$ pense la discipline comme une amère contrepartie de la démocratie : un partage - ou 
du moins une répartition - du contrôle social. Le roman policier «disciplinaire " pourrait alors figurer la participation du citoyen lecteur au pouvoir démocratiqueavec toutes ses tensions et ses contradictions ${ }^{13}$. Qu'en est-il sous le Troisième Reich ? Y a-t-il eu un Krimi de la dictature ou le genre policier a-t-il représenté un élément de pouvoir étranger au régime hitlérien et donc, paradoxalement, un point de résistance ? Par delà la simple étude d'influences, une analyse croisée du Krimi allemand et de la production étrangère permet d'interroger les spécificités et les interférences des pouvoirs démocratiques et totalitaires.

Notre propos est d'examiner la part d'« étrangeté » du Krimi sous le Troisième Reich, c'est-à-dire l'influence de la production étrangère sur le roman policier allemand, mais aussi son hétérogénéité par rapport aux discours en vigueur ainsi que l'altération des motifs traditionnels du genre. Le présent article s'appuie sur une quinzaine de romans parus entre 1933 et 1945 ainsi que sur de nombreux articles critiques publiés dans la presse spécialisée de cette période. Les auteurs et les maisons d'édition les plus célèbres et les plus prolifiques ont été privilégiés, mais une attention particulière a été accordée à quelques "marginaux ", susceptibles de produire des romans qui détonnent avec le discours de la RSK. L'article tend à démontrer que, entre rejet xénophobe et instrumentalisation idéologique, le Krimi a survécu à travers un exil imaginaire. Le genre est durablement marqué par la production anglo-saxonne-commercialement rentable, mais politiquement déconsidérée. À mesure que les relations internationales se raidissent, ces produits d'importation se font plus rares (1). Ils font place à une production nationale qui, comme le polar américain, se constitue à rebours de la tradition anglaise : est-ce à dire que le Krimi nazi s'apparente au roman hard-boiled? (2). Enfin, il apparaît que le Krimi s'est fait étranger à l'Allemagne et à son contexte policier (3): en effet, comment continuer à écrire des romans criminels alors que le crime a été officiellement vaincu ? L'étrangeté du Krimi se révèle aussi bien être une conséquence du pouvoir totalitaire qu'une de ses échappatoires.

\section{Un produit d'importation}

Le raz-de-marée de la littérature de masse, qui a débuté au XIX siècle et s'est propagé durant Weimar, n'a pas été endigué par la dictature. Certes, les parutions et les traductions de romans policiers ont tendance à diminuer, mais le Krimi a continué à prospérer sous le Troisième Reich. Sans surprise, cette production a largement été dominée par les œuvres anglo-américaines, avant que le régime ne mette un frein à cette mauvaise influence étrangère.

Il est assez difficile d'estimer-surtout au regard des sources officielles-le nombre de romans policiers parus en Allemagne durant la période nazie. S'appuyant sur la bibliographie de Schädel, Würmann $(2008,61)$ estime que les parutions entre 1933 et 1945 avoisinent les 3000 titres, le pic de production se situant dans les années 1937-1938 avec respectivement 385 et 447 nouveaux romans sur le marché. La plupart des grandes maisons d'édition possèdent une collection policière (Ullstein Verlag, Wilhelm Goldmann, Paul Zsolnay), et d'autres se spécialisent dans le domaine, notamment à Berlin avec l'Eden Verlag (et ses collections « Sammlung 50-Pfg-KriminalRomane » et «Ehrlichs Kriminalbücherei ») ou encore la Kulturelle Verlagsgesellschaft ("Iris-Kriminal-Romane »). Comptant parmi les plus beaux succès commerciaux de l'édition allemande à cette période, le Krimi de Georg von der Vring intitulé Die Spur im 
Hafen (La Piste du port), paru d'abord chez Scherl en 1936, puis chez Bertelsmann, se vend ainsi à plus de 350000 exemplaires.

11 En dépit de tels succès, la production anglo-saxonne se taille la part du lion. Jetant un regard sur la production des dernières années, un article du Bücherkunde déplore en 1939 : « Jusqu'à ces dernières années, le lectorat mondial a dû s'accommoder du fait que l'Angleterre détienne pour ainsi dire un monopole dans le domaine du roman policier $»^{14}$ (Ero, 154). Le terme de "monopole»-trahissant une ambition plus commerciale que littéraire-correspond à une certaine réalité. Le roman policier totalise plus de 600 traductions durant la période 1933-1944 (soit $14 \%$ des traductions publiées sous le régime nazi). Il constitue ainsi le genre littéraire le plus traduit du Troisième Reich (Sturge, 67). Pour les années 1933-1935, les œuvres anglo-américaines représentent près de $60 \%$ des Krimis paraissant en Allemagne. Des maisons d'édition comme Aufwärts (Berlin), Zsolnay (Vienne), Insel (Leipzig) et surtout Goldmann (Leipzig) en font un commerce juteux. Parmi les best-sellers, citons les œuvres d'Edgar Wallace-l'auteur le plus réédité de la période-publiées depuis les années $1920 \mathrm{chez}$ Goldmann. D'autres écrivains traduits avant 1933 continuent de jouir d'une belle popularité comme le montrent les fréquentes rééditions d'A. Conan Doyle ou E. Philips Oppenheim. Des auteurs plus récents investissent aussi le marché allemand: Agatha Christie-dont The Murder of Roger Ackroyd (Le Meurtre de Roger Ackroyd) est traduit dès 1926-voit ses romans connaître pas moins de 24 traductions différentes, y compris durant la guerre (Schädel, 162-6). Par les ventes qu'il génère et sa forte identité éditoriale (collections dédiées, parfois exclusivement constituées de traductions), le roman policier apparait comme une entreprise étrangère durablement implantée dans l'Allemagne nazie.

12 Encore faut-il déterminer de quel étranger il s'agit. Parmi les traductions, ce sont surtout les œuvres anglaises ou d'inspiration anglaise qui dominent. Les auteurs américains y sont beaucoup moins nombreux et ne se rattachent pas à l'école hardboiled: S.S. Van Dine (4 traductions durant notre période), Ellery Queen (6 traductions) et John Dickson Carr (7 traductions) sont parmi les plus connus. Comme en France, les polars à la Black Mask School ne seront (re)découverts que dans les années 1950. Dashiell Hammett et Raymond Chandler ne sont pas traduits sous le Troisième Reich. Parmi les rares parutions, Green Ice (Vivement mes pantoufles!) de Raoul Whitfield sort en $1931^{15}$, trois titres de Carroll John Daly entre 1933 et $1934^{16}$, de même pour Erle Stanley Gardner entre 1933 et $1939^{17}$. De fait, lorsque les critiques qualifient ce genre d'étranger, ils font surtout référence à la production anglaise.

13 Les États-Unis participent certes de l'imaginaire du crime en Allemagne, mais cette présence n'est pas directement littéraire. Sous le Troisième Reich, l'image de l'Amérique est fortement associée à l'industrie cinématographique : des stars comme Greta Garbo ou Marlene Dietrich sont particulièrement appréciées - et notamment par Goebbels ; les magazines allemands continuent d'entretenir le mythe hollywoodien par des reportages sur le quotidien de l'industrie filmique ; des films comme The BroadwayMelody of 1936 (Roy Del Ruth) ou les dessins-animés de Mickey Mouse constituent les plus grands succès de cette période. Les États-Unis sont un pays cinématographique (Gassert, 164-182). Depuis les années 1920, les articles de journaux se faisant l'écho de l'explosion du gangstérisme mais surtout des films comme Unterwelt (Underworld, J. von Sternberg, 1927) ont façonné l'image de New York ou Chicago comme métropoles du crime organisé. Après 1933, la production hollywoodienne représente une part non 
négligeable des films à l'affiche (Spieker, 337-47) ${ }^{18}$. À en croire leur durée moyenne de diffusion, les œuvres américaines sont en général plus appréciées par les spectateurs que les films allemands (id.). Certaines œuvres se taillent de beaux succès aussi bien publics que critiques: Ich bin ein entflohener Kettensträfling (I Am a Fugitive from a Chain Gang, M. LeRoy), sorti en 1933, reste 34 jours à l'écran et reçoit même les éloges du Völkischer Beobachter, journal du NSDAP. Dans l'ensemble, la représentation d'une Amérique criminelle s'appuie donc plutôt sur des références cinématographiques. Cela se ressent même dans les romans allemands. Si New York et Chicago fournissent le cadre habituel aux Krimis, il s'agit d'un décor de carton-pâte avec ses lieux obligés (Chinatown), ses personnages qui travaillent dans le cinéma (l'acteur félon tend à devenir un cliché) ou qui fréquentent assidûment les salles (Tobos, 1939) quand ils ne sont pas directement comparés à des stars comme Garbo (Rock, 1937, 19, 67 et alii). Ainsi, lorsqu'un des personnages de Gangster in der Prärie (Gangster dans la prairie) approche de New York, il fait route«en direction de la ville illuminée, dont les immeubles grandissaient à l'horizon comme dans un dessin-animé ${ }^{19}$ (ibid., 137).

L'imprécision de ces images de l'étranger reflète aussi le caractère diffus de leurs sources et leur tarissement progressif. L'univers criminel hollywoodien est loin d'être familier au grand public allemand. En effet, la censure est particulièrement sévère face aux films de gangsters. L'interdiction, en 1934, de Scarface de Hawkset la motivation largement diffusée - de cette décision donnent le ton : il n'est pas question de laisser passer des films qui glorifient le criminel, pourraient dévoyer les esprits fragiles et scandaliser les «bons » Allemands. Au fil des ans, les avis négatifs des deux chambres de censure cinématographique (la Filmprüfstelle et l'Oberprüfstelle) concernent un spectre toujours plus large d'œuvres "criminelles »: des classiques comme Manhattan Melodrama (W.S. Van Dyke, 1934) mais aussi des films plus légers comme The Thin Man (W.S. Van Dyke, 1934) ou Trouble in Paradise (Ernst Lubitsch, 1932) qui ont le tort de rire du crime. À cela s'ajoutent les individus dont les films sont «indésirables » du fait de leurs origines ou de leurs opinions politiques. Dès 1936, au sein du RMVP, circule une liste noire d'acteurs, réalisateurs et producteurs trop juifs pour être tolérés en Allemagne : y figurent entre autres Sylvia Sidney, Paul Muni, Edward G. Robinson, William Wyler, Michael Curtiz, Cecil B. DeMille, Ernst Lubitsch, Josef von Sternberg, Mervyn LeRoy, Archie Mayo-autant d'artistes figurant au générique des meilleurs crime movies. Quand Hollywood sort de sa réserve politique, la liste s'enrichit des noms des membres de la Hollywood Anti-Nazi League et du Committee of 56-James Cagney, Humphrey Bogart, Joan Crawford, Dashiell Hammett, et bien d'autres. Face à une censure toujours plus féroce, les compagnies américaines finissent par se retirer du marché allemand. D'autres avaient déjà déserté le Troisième Reich : c'est le cas de la Warner Bros. qui ferme sa filiale dès 1933-pour des motifs aussi bien politiques que commerciaux (Spieker, 90)-privant ainsi le public allemand des grands classiques du film criminel. Würmann $(2008,97)$ estime que 45 Kriminalfilme américains furent autorisés dans les salles du Troisième Reich. Malgré une hausse notable en 1938-1939, leur nombre tombe à 2 en 1940, dernière année où des productions américaines sont projetées en Allemagne.

15 Les traductions de romans policiers s'étiolent également. Leur part de marché diminue au fil des années, des censures et des restrictions. Elles profitent ainsi beaucoup moins de l'essor du Krimi en 1937-1939 que les romans allemands (Sturge, 154) : en 1938, on ne compte qu'une petite centaine de traductions contre près de 450 titres allemands. La chute s'accélère à partir de 1940: 150 nouveaux Krimis allemands sont publiés contre 
une dizaine de romans étrangers et, alors que l'on dénombre entre 50 et 100 nouveaux polarsles années suivantes, les traductions ne dépassent pas la dizaine (id.). Ce déclin ne s'explique pas seulement par la dégradation des relations internationales. La fin des années 1930 se caractérise par des pressions beaucoup plus fortes sur le genre policier. Entre autres causes, l'habituelle peur qu'inspire le Krimi, coupable d'inciter au crime: l'argument réapparaît au gré des faits divers et du zèle des fonctionnaires (Geyer-Ryan, 1987, 194) mais prend une certaine ampleur avec l'offensive policière menée contre la pègre et les asociaux à partir de 1937 (Wagner, 1996, 255-97). Des facteurs plus structurels expliquent cette mise au pas. Le 1er avril 1938, les diverses chambres culturelles du Reich doivent confier leurs tâches de contrôle politique au ministère de la Propagande. Autrement dit, la RSK remet ses prérogatives à la section VIII (Schrifttum, "Écrits ") du RMVP. Les organes de censure sont alors en voie de centralisation, finissant de mettre au pas les écrivains réfractaires-même si Goebbels ne parviendra finalement jamais à se débarrasser d'une organisation polycratique empêchant un parfait contrôle du domaine culturel. En tout cas, à la fin des années 1930, la dictature est assez fermement installée pour s'émanciper, occasionnellement, de la logique économique. Celle-ci n'est, au demeurant, pas étrangère au déclin de la littérature étrangère: le manque de devises entrave l'importation des produits littéraires en Allemagne (Adam, 233). L'action politique se trouve ici confortée par la situation économique.

Quoi qu'il en soit, les premiers titres apparaissent sur la Liste des schädlichen und unerwünschten Schrifttums ("Liste des publications nuisibles et indésirables») de 1938; les très populaires collections "Klings Erinnerungen » et «Klings Abenteuer » de Werner-Dietsch-Verlag (Leipzig) sont interdites le 16 juillet 1938 ; à partir du 1er juin 1939, les séries romanesques doivent bénéficier d'une autorisation préalable ; en 1940, Goebbels inscrit 14 séries de romans criminels, d'aventure et de western sur la Liste der für Jugendliche und Büchereien ungeeigneten Druckschriften («Liste des imprimés inappropriés pour la jeunesse et les bibliothèques »). Les pressions s'accentuent sur des éditeurs de littérature populaire comme W. Goldmann Verlag (Barbian, 242-44). Dès 1934, la RSKexige qu'il soumette ses livres à un examen préalable comme la Beratungsstelle für Unterhaltungsliteratur ${ }^{20}$, l'exige de tous les éditeurs de ce secteur en juillet 1935. Puis, la RSK refuse, "par principe ", à Goldmann l'autorisation d'acquérir de nouveaux droits de traduction sur les romans policiers anglais et américains. Menacé d'exclusion de la RSK, Goldmann s'exécute, sans que sa situation ne s'en trouve améliorée: en 1940, l'ensemble des œuvres d'Edgar G. Wallace est interdit. Plus largement, ce sont toutes les traductions qui pâtissent de l'entrée en guerre. Depuis 1935, les éditeurs devaient demander à la RSK une autorisation préalable à l'achat des droits étrangers. En 1941, un article de la Weltliteratur ${ }^{21}$ énonce des règles strictes pour choisir judicieusement les œuvres qui méritent d'être traduites. L'injonction « Nous refusons tout auteur qui tient des propos incendiaires contre nous ${ }^{22}$ (cit. in Adam, 231) pèse ici particulièrement lourd. Finalement, avec l'entrée en guerre des États-Unis, Himmler et le RMVP interdisent la diffusion et la circulation de toute publication américaine.

17 Le «monopole » anglo-américain sur la littérature policière serait-il une invention de la propagande nazie ? Cette hégémonie est sans doute exagérée, mais certainement pas fantasmée. L'importance de la production étrangère est plus qualitative que quantitative. De l'avis général, les crime novels fournissent l'étalon de mesure du Kriminalroman. Ainsi, un article de Die Literatur dressant l'état des lieux du roman 
policier, fait l'éloge d'Austin Freeman, Sax Rohmer, Edgar Wallace tandis que Philip MacDonald et S.S. Van Dine sont cités comme des écrivains prometteurs-et le journaliste de déplorer l'absence d'auteurs de cette envergure en Allemagne (Schickert 247, cit. in Würmann, 2008, 89). Quelques années plus tard, un critique reconnaît encore : « Le roman criminel anglais a toujours été un exemple pour le roman allemand ${ }^{23}$ (Thielke, 43). La production hollywoodienne sert, quant à elle, d'exemple au film allemand. En janvier 1938, un article de la revue Der deutsche Film sur la précision du travail dramaturgique loue la finesse des dialogues de After the Thin Man (W.S. Dyke, 1936, scénario de D. Hammett). Quelques années auparavant, le Völkischer Beobachter du 18 mars 1933 considérait I Am a Fugitive from a Chain Gang comme " un film qui peut servir d'exemple quant à la manière de présenter des événements de manière concise, logique et objective $»^{24}$ (cit. in Spieker, 130). Même quand le film américain devient indésirable, les journaux encensent les productions allemandes capables de rivaliser avec Hollywood ${ }^{25}$. Les États-Unis restent la référence cinématographique-à défaut d'être un modèle littéraire. On peut toutefois supposer que les romans américains profitent à quelques "happy fews ». En effet, si les traductions sont interdites, les œuvres en langue originale sont toujours autorisées. Plusieurs sources attestent que, au moins jusqu'en 1940, les pulps et les polars américains étaient disponibles en librairies (Adam, 59; Würmann, 2004, 182). De plus, une maison d'édition comme The Albatros (Hambourg-Leipzig), spécialisée dans les textes anglophones, continue de proposer les œuvres d'Agatha Christie, mais aussi The Maltese Falcon de Hammett en 1932 ou The Postman Always Rings Twice de James M. Cain en 1939. Il est certes difficile d'évaluer la diffusion-et les retombées-de ces œuvres. Même parmi les américanistes, des voix s'élèvent contre le polar: Friedrich Schönemann-professeur de l'unique chaire de littérature nord-américaine sous le Troisième Reich-rédige des articles incendiaires contre les nouveautés policières de The Albatros qu'il considère comme issues de la "mauvaise » Amérique, d'une culture décadente, judaïsée, qu'il oppose à l'Amérique blanche et protestante (Gassert, 239-40). Cependant, le polar hard-boiled a aussi pu toucher le roman policier allemand dans la mesure où de nombreux traducteurs ou simples anglophones étaient eux-mêmes auteurs de Krimis. C'est le cas, par exemple, de C.V. Rock (Kurt Walter Roecken, 1906-1985), auteur de plusieurs dizaines de romansqui se déroulent le plus souvent à Chicago ou à New York -, traducteur et qui, toute sa vie durant, a entretenu une relation épistolaire avec Erle Stanley Gardner (Weigand, 189).

18 Au-delà des supputations, un élément pourrait résumer l'hégémonie étrangère sur le genre policier: les pseudonymes anglo-américains que se choisissent les auteurs allemands, tels C.V. Rock mais aussi Robert Arden (Robert Heymann) ou Ferry Rocker (Lena Eschner). Ce détail est assez révélateur. Il correspond à un horizon d'attente des lecteurs et résulte d'une logique commerciale selon laquelle les bons romans policiers sont exclusivement anglophones. Cette pratique n'est pas spécifique au marché allemand, mais elle détonne avec l'idéologie en vigueur. Alors que les noms pompeusement germaniques ou les patronymes chrétiens germanisés sont très prisés sous le Troisième Reich (Klemperer, 115), opter pour un pseudonyme anglo-saxon est une félonie : l'auteur, cherchant le succès dans la littérature de masse, s'est vendu à l'étranger. On comprend mieux pourquoi ce détail éditorial attire fréquemment les foudres de la critique. Erich Langenbucher s'indigne ainsi dans Die Buchbesprechung: "On sait que beaucoup de romans policiers étrangers ont connu un grand succès et, certes, qu'ils ont en partie servi de modèles à des auteurs qui n'étaient pas des 
incapables, d'où cette incompréhensible allégeance que l'on exploite à outrance... $»^{26}$ (38).

19 Le roman policier perdure en tant que littérature étrangère sous le Troisième Reich L'horizon d'attente place ce genre-sa forme, sa structure-sous le signe du detective novel à l'anglaise tandis que la présence américaine, quand elle ne se traduit pas par des images stéréotypées, est assez disparate. Le roman policier n'en est pas moins affecté par le nouveau régime. À mesure que la dictature se fait plus féroce, la production se fragilise. Les sources d'inspiration étrangères se tarissent progressivement et les auteurs allemandsdoivent suppléer à cette pénurie. La concurrence extérieure supprimée, ils peuvent continuer à produire des ersatz de Sherlock Holmes-à moins qu'une telle disparition ne permette l'émergence d'un roman policier national. C'est du moins ce que souhaite Langenbucher en une menace à peine voilée et des termes empreints d'idéologie Blut-und-Boden : « Il est temps que ceux qui se prétendent auteurs de romans criminels changent radicalement. En effet, ils ne pourront plus longtemps récolter les fruits d'une telle engeance $»^{27}(41)$. Le Troisième Reich fournit-il le terreau d'un nouveau Krimi ou le sol allemand est-il désormais impropre à l'épanouissement du roman criminel?

\section{Vers un Krimi national-socialiste?}

Conformément à la logique de concurrence et de préférence nationale qui doit régir le marché du livre sous le Troisième Reich, on attend du roman policier qu'il se réforme et supplante la production anglo-saxonne: "Il ne sera pas difficile de faire lever le camp à la camelote d'un Edgar Wallace et de la remplacer par ce que souhaitent depuis longtemps en silence de nombreux lecteurs: le bon roman policier allemand $»^{28}$ (Brunner-Ebersperg, 15), écrit un critique en une phrase mêlant termes guerriers et commerciaux. Cependant, qu'un article de 1942 emploie encore le futur laisse douter du succès de l'entreprise. A-t-on réussi à "nationaliser » un genre qui semblait irrémédiablement étranger? Un "bon roman policier allemand" était-il même possible selon les critères du Troisième Reich?

\section{Un roman criminel policier}

Le genre policier est considéré par certains comme une expression propre aux peuples anglo-saxons. Helmut Günther (1940-1941), par exemple, le décrit comme irréconciliable aussi bien dans ses motifs que dans sa forme avec l'esprit germanique. Le Krimi, produit d'une civilisation étrangère, agit comme un corps allogène au sein de l'Allemagne. Avec la guerre, l'influence anglo-saxonne devient rapidement synonyme de propagande ennemie. Ainsi, le RMVP, dans une note confidentielle du 16 avril 1940 à la RSK, rappelle qu'il convient d'épurer la littérature des "influences ennemies" (feindliche Einflüsse) et notamment la littérature criminelle qui «fait clairement l'apologie des institutions et du mode de vie anglais ou qui met en avant le travail de la police anglaise $»^{29}$ (cit. in Geyer-Ryan, 1987, 198). Pour la Großdeutsches Leihbüchereiblatt, la littérature populaire doit prendre en compte la nouvelle donne internationale : « Elle doit être émancipée de toute influence hostile à l'État et, en ce qui concerne le roman policier, elle doit s'abstenir de toute référence à Scotland Yard et à la police anglaise $»^{30}$ (Losch, 42). Plus encore qu'à l'âme germanique, ce sont aux institutions et autorités 
allemandes que les romans policiers portent ombrage. Le Krimi doit se choisir de nouveaux héros : «Un Sherlock Holmes allemand, ce ne serait pas mal non plus! » $^{31}$ (Ero, 1939, 155), peut-on lire dans le Bücherkunde. Cette revendication est savoureuse : l'article exclut toute imitation de la figure du détective-invention spécifiquement anglaise ${ }^{32}$-pour ensuite se référer au personnage de Conan Doyle, reconnaissant ainsi l'autorité anglo-saxonne en la matière. En tout cas, ce refus désigne clairement le contre-modèle du roman policier allemand. Comme pour le polar hard-boiled américain, le détective anglais constitue un repoussoir. Le Krimi s'oppose moins à Sam Spade qu'à Sherlock Holmes.

S'il s'émancipe de l'influence anglo-saxonne, le roman policier peut se réformer et servir le régime-c'est du moins ce qu'arguent ses défenseurs, tel Edmund Finke, luimême auteur de Krimi et proche du Parti : «Au fond, le roman criminel, d'un point de vue moral, ne traite de rien d'autre que [...] de la guerre des forces de l'ordre contre le chaos déchaîné des bas-fonds ${ }^{33}$ (Finke, 1939, 333). De ce fait, le roman policier peut remplir un rôle éducatif ("erzieherische Aufgaben») s'il met en avant le policier héroïque plutôt que le vil asocial. Langenbucher abonde dans ce sens quand il demande : "La police allemande ne mérite-elle pas d'être la véritable héroïne de ces livres? $»^{34}(41)$. Ce changement est déjà largement amorcé en 1939. Il accompagne une action policière plus large contre la pègre allemande qui, depuis 1933, voit se succéder razzias et internements préventifs : on estime qu'environ 70000 "asociaux» sont arrêtés par la police criminelle du Troisième Reich (Wagner, 1996, 2). En outre, la Kriminalpolizei est modernisée, centralisée et dotée de nouveaux pouvoirs. La propagande quotidienne vante son efficacitéet la production littéraire s'en fait l'écho.

Contrairement aux Krimis des années 1920, où la défiance envers les serviteurs de la République était prégnante, la majorité des romans parus après 1933 prennent le policier pour héros. C'est le cas notamment de Der Tod fuhr im Zug (La Mort voyage en train)écrit en 1944 par Axel Alt-pseudonyme de Wilhelm Ihde, directeur (Geschäftsführer) de la RSK-en collaboration avec le service de relations publiques de la police du Reich. Cette enquête-basée sur des faits réels et, comme l'affirme le soustitre, sur les procès-verbaux de la Kripo-se donne pour mission de présenter la police allemande dans toute son efficacité. Toutefois, se fonder sur les dossiers et archives n'engage aucunement à l'objectivité. Le narrateur omniscient s'intéresse exclusivement au travail des enquêteurs et donne quelques maigres indications au cours du récit quant à l'identité du coupable-relativisant ainsi l'opacité du mystère tout en refusant d'explorer plus avant la personnalité du criminel : ce sont en effet des « détails qui sont certes importants pour les criminalistes et quant aux motivations du coupable, mais qui pour nous sont négligeables dans le cadre de cette présentation $»^{35}$ (217). En revanche, une telle retenue n'est plus de mise quand il s'agit de présenter le commissaire menant l'enquête : on pénètre dans son intimité, ses passe-temps, ses pensées et l'on découvre un homme débonnaire mais tenace, un professionnel consciencieux au regard et au cœur d'artiste-s'octroyant ainsi les attributs traditionnels d'un assassinat conçu comme l'un des beaux $\operatorname{arts}^{36}$. La narration-soit par la focalisation, soit par les descriptions-penche en faveur du gardien de l'ordre, homme aux goûts simples et se satisfaisant du devoir rempli, assez proche du lecteur pour pouvoir servir d'exemple. Si l'homme est simple, sa fonction doit être trépidante pour s'orner d'héroïsme. Sous le Troisième Reich, l'opération de police est une scène très prisée des films et des romans. En effet, l'action mouvementée est un ingrédient traditionnel de la culture populaire 
alors que le dynamisme est au fondement rhétorique de la LTI (Klemperer, 291-93). La production est donc facilement gagnée par des policiers combattant le crime au pas de charge. Les romans culminent souvent par des descentes de police parfaitement coordonnées-par exemple, dans Gangster in der Prärie, où affleure une critique des systèmes ankylosés par le respect du fédéralisme (Weimar ou les États-Unis) (Rock, 42, 82). L'Allemagne, désormais unifiée, est entièrement contrôlée par une police rapide et efficace, comme le montre le film Großalarm (Chasse à l'homme,Georg Jacoby,1938) dont le titre annonce le véritable sujet : l'action menée par la Kripo.

Inversement, son adversaire est le plus souvent relégué à l'arrière-plan ${ }^{37}$. Dans Zehn einwandfreie Alibis (Dix Alibis irréprochables)de Finke, le criminel disparait presque du Krimi. Durant tout le roman, on recherche un cambrioleur qui n'apparaît jamais au cours de la narration. Il n'est jamais décrit, mais seulement mentionné dans les conversations et les rapports des inspecteurs : le criminel est tout entier placé sous le discours de la police. Cette éclipse n'est pas toujours aussi complète mais, dans la majorité des romans, le truand devient falot. Ses actions ne peuvent susciter l'admiration. La Reichsschrifttumsstelle demande d'éviter les actions trop trépidantes, pouvant exciter les désirs d'aventure ${ }^{38}$. Plutôt que de grands crimes, le Krimi doit traiter de forfaits communs : « Les faux passeports, le trafic de devises, l'enlèvement d'enfant, les vols de voiture ne sont-ils pas des problèmes sérieux ? ${ }^{39}$, demande Langenbucher $(1939,41)$ pour fustiger les intrigues mirifiques. Les criminels en sont réduits à commettre de petits délits qui ne leur font pas honneur. Dans Zehn einwandfreie Alibis, on prend soin de préciser que la recette dérobée n'est pas importante. Dans Gangster in der Prärie, les truands sont trop indisciplinés pour réussir un gros coup. Le roman policier remplit ainsi la fonction dont parlait Finke, à savoir montrer que « le crime [...] ne révèle rien d'autre qu'imbécillité et paresse fourvoyée car le criminel ne parvient régulièrement à un but médiocre que par les chemins les plus escarpés et prend un risque complètement disproportionné par rapport au "gain" que lui rapporterait éventuellement le crime $»^{40}$ (Finke, 1939, 333).

Cette récupération policière du Krimi n'est pas sans rappeler le retournement que connaît la culture de masse américaine des années $1930^{41}$. Le gouvernement déclare la « War on Crime » le 17 juin 1933 après la fusillade de Kansas City : deux agents fédéraux abattus et une opinion publique, déjà exaspérée par le gangstérisme des années 1920, qui appelle à des mesures drastiques. Sous la direction de Homer Cummings, le département de la Justice est réorganisé, les pouvoirs de l'agent fédéral sont accrus, la juridiction du Bureau est élargie et les G-Men deviennent le nouveau modèle policier. Le gangster est renversé de son piédestal où l'ont installé la littérature et le cinéma. On lui oppose un rival médiatique : J. Edgar Hoover, le directeur du Bureau lui-même. Avec l'aide de quelques publicitaires, le petit homme se transforme en pilier de la lutte contre le crime. Il encourage à prendre l'agent fédéral pour héros ${ }^{42}$ tandis que la Crime Records Division du Bureau aide à produire des émissions radiophoniques, des bandesdessinées, des magazines pulp, et des films. Parallèlement, certains studios et acteurs, coutumiers des films de gangster, changent leur fusil d'épaule : c'est le cas de la Warner avec ses films où les policiers sont désormais incarnés par des stars tels James Cagney et Edward G. Robinson ${ }^{43}$. Ici comme en Allemagne, l'industrie culturelle sert une campagne sécuritaire: la gloire du policier, désormais surpuissant, éclipse le personnage du criminel. 
26 La comparaison ne doit cependant pas engendrer d'amalgames. Certains motifs semblent converger à travers le durcissement du pouvoir policier dans les deux aires, mais celles-ci ne relèvent pas de la même logique de pouvoir. L'autoritarisme latent de la «War on Crime » ne peut être assimilé à la dictature dans laquelle l'Allemagne a basculé ${ }^{44}$. Des exemples très simples en témoignent. La production culturelle touchant aux G-Men en appelle constamment aux instances démocratiques (élections, chambres parlementaires) pour doter la police de nouveaux pouvoirs ou au peuple américain afin qu'il aide l'agent fédéral dans sa croisade (les enfants s'inscrivent dans des clubs de détective ; les adultes sont priés d'ouvrir l'œil pour arrêter les Public Enemies No 1 que Hoover désigne à la presse). Dans l'Allemagne nazie, en revanche, le pouvoir policier ne se partage pas. Un film comme Großalarm est sans ambiguïté à ce sujet. Des livreurs de journaux mettent en place un système de surveillance qui n'est pas sans rappeler celui de la pègre dans $M$ de Fritz Lang (M le maudit, 1931) : sous couvert de faire leur travail, ils peuvent suivre les criminels. Leur filature échoue lamentablement. C'est seulement lorsqu'ils préviennent la police qu'un impressionnant dispositif quadrille tout le pays et élimine prestement les truands. Ni la pègre ni le peuple ne peuvent rivaliser avec le pouvoir policier.

Ce monopole étatique de la surveillance se traduit aussi par un personnage de détective dévalorisé. En effet, on fustige l'utilisation des officines privées. Même si Langenbucher s'indigne encore en 1939 («Dans de très nombreux cas, à la police, s'oppose le détective, bien plus malin que les gardiens de l'ordre $»^{45} ; 40$ ), la RSK se prononce dès 1935 sur la question, après ses journées de travail sur la littérature populaire : « il n'est plus possible que le détective privé demeure le héros de l'intrigue ${ }^{46}$ (Anonyme, 1935, 1015). Le détective privé apparaît rarement dans le Krimi du Troisième Reich. Quand il ne travaille pas en étroite collaboration avec les policiers-au point de mener les interrogatoires dans leurs bureaux comme dans Mord in Pittsburg (Meurtre à Pittsburgh, Tobos, 1939)-il est tourné en ridicule. Dans Zehn einwandfreie Alibis de Finke, les détectives ne servent qu'à colporter des ragots conjugaux. Dans une nouvelle de Michael Zwick (1934, 41 sqq.), intitulée «Der Detektiv ", c'est le genre du roman de détection lui-même qui est parodié grâce à un détective retraité, sorte de Don Quichotte du Krimi. Pipe à la bouche, coiffé d'un chapeau grotesque, il enquête sur une montre égarée. Comme dans les classiques anglais, le détective disparaît juste avant la résolution de l'intrigue : la montre est retrouvée par la femme de ménage et le ridicule Sherlock Holmes revient en piteux état, rossé par des villageois qu'il a accusés.

La nouvelle de Zwick est amusante, mais l'éclipse du détective n'en révèle pas moins des mécanismes idéologiques importants. Encore une fois, il s'agit d'une confiscation étatique des dispositifs disciplinaires. Le détective américain peut refléter une défiance face aux institutions ainsi qu'une certaine tradition libérale, réticente à un monopole $\mathrm{du}$ pouvoir policier. Ces personnages ont prospéré car ils servaient des causes individuelles que les pouvoirs publics ne pouvaient contenter. Ils pouvaient dénoncer les vices du système judiciaire, la corruption des édiles et l'impunité des puissants. Sous le Troisième Reich, cette figure est devenue inutile, voire dérangeante. La corruption et les lacunes policières ont disparu. Les causes individuelles n'ont plus lieu d'être, car le particulier doit se sacrifier à l'intérêt général, incarné par l'État ${ }^{47}$. Au demeurant, la séparation des individus et des institutions n'a plus sa place dans la communauté populaire du Troisième Reich: «La police allemande n'est pour nous ni un service administratif ni l'instrument $d u$ pouvoir étatique en soi seulement, mais la 
représentation, dans son domaine, de la communauté raciale allemande $»^{48}$, écrit Brunner-Ebersperg (14) pour définir le nouveau Krimi allemand.

L'accaparement du pouvoir reflète aussi une logique propre au système totalitaire : investir les sphères privées dans leurs moindres recoins. Cela ne concerne pas simplement les systèmes de contrôle et le refus de toute concurrence policière, mais aussi les récits, les divertissements liés à ces systèmes. Selon Hannah Arendt (46), les régimes totalitaires récusent toujours la séparation de la sphère sociale et du divertissement; ils refusent de laisser subsister entre les sujets certains liens communautaires (familiaux, culturels) qui ne sont pas encadrés politiquement. De là, la promotion des livres de sciences politiques ou raciales et des films exposant les vues du régime. En revanche, un article se présentant comme une « étude psychologique » du lecteur de Krimi lui reproche de se livrer non pas à une lecture mais à une " occupation " (Beschäftigung) qui l'isole du reste de la communauté raciale (Eichberg). Et pourtant, la sphère du roman policier n'est pas hermétique : le remplacement du détective privé par le policier est bien le signe d'une prise de contrôle de l'imaginaire romanesque.

\section{« Hmm, bon, alors on va déroger à la règle encore une fois ! »49 : Krimi hard-boiled ou Weltanschauung?}

Il faut garder à l'esprit cette différence entre détective américain et policier allemand si l'on veut comparer le Krimi au polard'outre-Atlantique ${ }^{50}$. Leurs écarts ne prennent pas place dans les mêmes champs idéologiques et discursifs. Leurs transgressions des normes judiciaires et littéraires n'ont pas les mêmes significations-ne serait-ce que parce que les « dérapages » d'un détective privé deviennent plus problématiques quand ils sont le fait d'un représentant de l'État et prennent paradoxalement un statut de normes.

Comment comprendre les entorses à la loi commises par les enquêteurs? Aux ÉtatsUnis,en 1927, Carroll J. Daly reconnaît, dans un entretien à Black Mask, qu'ellessont une réponse aux carences juridiques : " the law is too cumbersome, too full of loopholes to be of much use " (cit. in Nolan, 39). Aux lois trop rigides pour s'adapter au quotidien, trop larges pour ne pas être détournées, le détective oppose une justice pratique et indépendante. Le détective Race Williams, affirme ainsi : "Right and wrong are not written on the statutes for me » (Daly, 1981, 12). De là, ses dérapages, ses mensonges et quelques passages à tabac: la Justice ne peut triompher qu'avec les mains sales et le détective hard-boiled a des allures de mauvais garçon $^{51}$. Le policier allemand s'en rapproche également. Dans Gangster in der Prärie, l'adjoint de Chappell, Bobby, est un justicier dont la scélératesse est une réponse efficace au crime. Gangster repenti, il n'est pas tout à fait amendé: «Bobby n'avait cependant pas encore abandonné ses habitudes de gangster $\aleph^{52}$ (46). Il fréquente encore les bas-fonds, récoltant brutalement les informations. À la fois policier et malfrat, il commet d'innombrables bavures, excusées au nom de la loi. De même, les agents de Mord in Pittsburg ne se privent pas de tabasser les criminels. Cette pratique est courante dans les Krimis et largement acceptée par les héros et les officiers de police: «Au début, le patron du bar ne voulait pas se mettre à table, mais finalement-tu connais mes hommes, hein-il s'est laissé convaincre $\aleph^{53}$ (Tobos, 144), dit avec indulgence l'inspecteur Kennedy au détective Buckley. Chez Rock, lorsque Bobby admet avoir procédé à un interrogatoire musclé 
("C'était bougrement illégal!»), son supérieur ricane et lui répond: «Ce que Durop a fait était beaucoup plus illégal encore! (Rock, 1937, 238) $)^{54}$. Il n'est pas nécessaire de respecter la loi face à ceux qui la violent.

Cette mise hors-la-loi, c'est-à-dire le refus d'une quelconque protection judiciaire pour les criminels, est le résultat d'une logique propre au Troisième Reich et qu'Ernst Fraenkel a analysée dans The Dual State (1941): à l'État de norme (Normenstaat) se superpose un État de prises de mesures (Maßnahmenstaat). Les dirigeants politiques (par des décrets, des « mesures »), mais aussi la police (en corrigeant des décisions de justice jugées trop clémentes ou en adaptant librement la procédure à la situation) et les juges (par des interprétations très larges des textes) introduisent de l'arbitraire dans un système légal dont la plupart des articles n'ont pas été modifiés depuis Weimar. C'est que les textes n'ont plus besoin d'être formellement transformés. Cette transgression de la lettre au nom de l'esprit des lois-c'est-à-dire la négation du système juridique au nom d'une efficacité policière-est partie intégrante de la conception de la justice sous le Troisième Reich. Werner Best (1903-1989), idéologue de la police nazie, explique que la Verordnung du 28 février $1933^{55}$ a constitué un tournant décisif qui a bouleversé la conception de l'État (Staatsauffassung)et de la loi (Best, 17): «Dès lors, toutes les dispositions légales qui s'en écartent doivent être considérées comme amendées dans le sens de cette nouvelle conception - et ce sans que chacune des anciennes lois ne soit abrogée ou amendée de manière formelle $»^{56}$ (ibid., 19). Les responsables politiques, policiers et juridiques s'accordent ainsi avec les personnages de Krimi : la justice ne se trouve pas dans les livres de lois, mais dans l'action. Le juriste Walther Sommer (1893-1946) résume en 1937: «Une administration saine n'a rien à voir avec les paragraphes de loi $»^{57}$ (cit. in Stolleis, 25) et, la même année, l'inspecteur Reg Chappell s'écrie dans Gangster in der Prärie: «Habituellement, je respecte la procédure! Mais c'est terminé maintenant ! Dorénavant, ça va mitrailler ! ${ }^{58}$ (149).

33 La loi et la manière de l'administrer sont désormais fonctions de l'idéologie nazie ${ }^{59}$-ou plus exactement de la Weltanschauung (grossièrement: "vision du monde »), qui désigne une compréhension intuitive, à laquelle participe l'essence de l'observateur, par-delà la froide raison positiviste. Héritage détourné de la Lebensphilosophie, cette notion est une pierre angulaire du discours nazi et lui a souvent servi à attaquer la Vernunftrepublik, la raisonnable République de Weimar-à laquelle on oppose le sentiment de la communauté et l'expérience vécue (Erlebnis). On la retrouve dans les critiques adressées au roman policier, genre où la logique prime sur l'âme et le sentiment métaphysique ${ }^{60}$. Même Edmund Finke considère la ratiocination comme un attribut criminel: "Quand il dépeint un gentleman criminel, l'auteur de Krimi veut représenter le danger d'un intellectualisme irresponsable qui enlève au monde sa spiritualité et le transforme en terrain de jeu de froides passions sans amour $\aleph^{61}(1939$, 333). Cette critique de la raison va évidemment à l'encontre des règles édictées par S.S. Van Dine en 1928 («The detective story is a kind of intellectual game ») et par le London Detection Club en 1930. Encore une fois, le rejet du modèle anglais fait converger Krimis allemands et polars américains. Distinguer le raisonnable roman à l'anglaise et la fiction hard-boiled est un lieu commun de la critique ${ }^{62}$. Plutôt que ses petites cellules grises, le détective américain utilise ses poings. Le Krimi prend également ses distances avec le mode de détection anglais: outre la violence, il s'agit de juger presque instinctivement la personnalité des suspects. Finke demande que les auteurs de romans policiers fassent surtout preuve d' "un cœur sensible et chaleureux, une véritable connaissance des hommes, acquise par l'expérience $»^{63}(1939,334)$. Les enquêteurs 
allemands collectent peu d'indices et ne cherchent pas en tirer de stupéfiantes déductions. Ils procèdent plus volontiers par des interrogatoires qui mettent à nu la véritable nature des suspects. Ils se fient aux réactions involontaires, aux regards et aux tressaillements que provoquent leurs questions et leurs intimidations. Ainsi, dans Mord in Pittsburg, le détective n'inspecte les lieux du crime qu'au bout des deux-tiers du roman, et n'y trouve rien d'intéressant (213). En revanche, son adjoint s'en remet à ses impressions quand il interroge un truand: "Il sent d'instinct que ce type lui a dit la vérité $\aleph^{64}$ (245). De même, le Reg Chappell de C.V. Rock mène sans ambages ses interrogatoires; plutôt que de déduire, il pose des questions brutales pour voir (schauen) la réaction des suspects : «Chappell aimait ce genre de spectacle [Schauspiele]. Il était d'avis que des effets d'annonce aussi dramatiques pouvaient être incroyablement instructifs. Et l'expérience lui donnait raison $\|^{65}(1937,177)$. La vision, le ressenti et l'expérience remplacent la ratiocination; la loupe du détective disparaît au profit de la Weltanschauung: "Je suis simplement capable d'ouvrir les yeux un peu plus grands ${ }^{66}$ (ibid., 237), dit Reg quand il se compare aux autres enquêteurs.

Cependant, le refus de la ratiocination des Krimis et du polar n'est pas sous-tendu par la même vision du monde. Dans les romans hard-boiled (chez Hammett et Chandler notamment $)^{67}$, l'enquête laisse entrevoir l'absurdité d'un monde vicié, sans rétablir un ordre et une vérité stables. Les forfaits et leurs récits s'encastrent, et si le détective résout une affaire, il ne clôt jamais l'histoire du crime-au contraire, il la nourrit en soulevant de nouvelles questions et en donnant des faits des versions variant selon les intérêts de chaque protagoniste. Le détective pointe le mensonge et perpétue «la puissance du faux » (Deleuze, 117) : à la fin du roman hard-boiled, quand bien même les coupables sont arrêtés, notre jugement reste en suspens. Cela n'est pas le cas dans les Krimis, qui cherchent à installer une certitude. Quoique la Weltanschauung des nazis ne soit jamais clairement définie, elle se veut limpide, se fondant sur le sang et la volonté du Führer, s'articulant autour de quelques mots-clés. On vise ainsi une "vérité organique "-notion introduite par Rosenberg dans le Mythe au xxe siècle-qui ne consiste pas en un savoir logique, mais germe inconsciemment dans l'individu et se trouve « au centre mystérieux de l'âme du peuple et de la race » (Klemperer, 141). On peut ici reprendre la typologie habituelle du genre policier (Todorov, 1971). Si le roman classique procède par rétrospection et abduction, si le polar américain consiste en une prospection et une reconstruction du monde inspecté, le Krimi du Troisième Reich se fonde plutôt sur la persuasion: l'enquêteur est certain de ses impressions, qui correspondent en fait à une juste Weltanschauung. Contrairement au polar, le doute n'est plus permis et le Krimi doit être catégorique : il faut, à la fin, séparer le bon grain de l'ivraie. Alors que le détective américain découvre une société grouillante de malfrats, l'auteur germanique y répugne naturellement : « C'est à contrecœur que nous contrefaisons un monde où le crime peut, pour ainsi dire, advenir derrière chaque porte $»^{68}$, écrit un critique dans Die Literatur (Anonyme, juillet 1939, 583). Plus que dans le roman policier classique, il faut que l'ordre social soit indubitablement rétabli.

Cette conviction s'appuie sur certains traits formels du Krimi. Premièrement, le souci de ne laisser aucun élément dans l'ombre incite l'enquêteur à tout expliciter: le moindre fait-même quand il est facilement inféré par le lecteur-est sujet à commentaire. Les actes, gestes et pensées de chacun sont absolument transparents. Ainsi, dans Mord in Pittsburg de Tobos, un gangster s'étonne que l'adjoint du détective se rende dans un bar interlope, joue aux cartes avec lui tout en reconnaissant avoir reçu l'ordre de le surveiller. Le criminel subodore un autre piège mais le détective affirme en toute 
franchise : «T'es une canaille bien soupçonneuse [...]. Quand j'ai été embauché chez lui, il [mon chef] a posé comme condition que je dise toujours ce que je voulais entreprendre contre mon adversaire $»^{69}(96)$. Et, effectivement, l'intrigue ne révélera aucun double-crossing de la part du détective. On est bien loin des stratagèmes déployés par Beaumont dans The Glass Key de Hammett ou de l'opacité du « style objectif » décrit par C.-E. Magny (44-61); les personnagessemblent ne plus rien avoir à cacher. Cela se vérifie par la pratique récurrente de l'aveu dans le Krimi duTroisième Reich. Pour un pouvoir qui veut s'imposer absolument, sans les objections des criminologues ou les indulgences d'un juge, l'aveu constitue une pièce maîtresse de son argumentaire, la preuve absolue. La plupart des romans se terminent ainsi par une confession de criminels plutôt que par la résolution du crime au moyen d'indices. Plus encore, certains personnages se rendent d'eux-mêmes à la police pour leur faire des révélations, comme dans Zehn einwandfreie Alibis de Finke ou Die Opiumhöhle von Cayenne de Brock. Si, dans ce dernier roman la collaboration avec la police est toujours récompensée, dans Mord in Pittsburg, toute l'intrigue naît de la réticence d'un homme d'affaires à informer les forces de l'ordre : «Si vous [...] n'aviez pas craint de rendre cette affaire publique, l'affaire du meurtre Sprigg n'aurait jamais existé $»^{70}(300)$, lui dit le détective à la dernière page du roman. De gré ou de force, les personnages du Krimi sont enjoints à parler.

Deuxièmement et parfois à rebours de ces explications bavardes, le Krimi cherche à établir la certitude de l'enquête par une simplification de l'intrigue et du langage. Le genre policier a été souvent attaqué pour ses énigmes tortueuses et ses phrases alambiquées, syntaxiquement incorrectes et remplies de mots étrangers-le mensonge des unes se reflétant dans les autres. Certains critiques y trouvent même la cause de la déficience allemande dans ce genre littéraire : la langue allemande-langue de vériténe se prête pas à ces artifices typiquement anglo-saxons ${ }^{71}$. Or, dans les discours nationalistes allemands, la langue et l'esprit d'un peuple sont intimement liés; l'avilissement de l'un entraînerait la décadence de l'autre. Pour ses détracteurs, le Krimi a largement contribué à déformer la langue allemande ${ }^{72}$, quand ils ne parlent pas franchement de viol maternel: "dans beaucoup de ces productions, notre langue maternelle est violée à chaque page. $»^{73}($ Diehl, 24). Il s'agit de mettre un terme à ce crime linguistique. Dans son discours d'inauguration de la deutsche Haus à Munich, après avoir déploré que les concepts obscurs et l'amphigouri aient longtemps engourdi l'esprit du peuple, Hitler assène : « Être allemand veut dire : être clair » ${ }^{74}$ (cit. in Gilman, 174). De même, Georg von der Vring, l'auteur du très prisé Spur im Hafen, affirme dans un discoursau titre programmatique ("La simplicité dans la littérature ») que " tout ce qui est beau est simple $»^{75}$ tandis que Langenbucher en appelle à des romans 《linguistiquement et littérairement décents ${ }^{76}(40)$. Plus concrètement, ces injonctions se traduisent par une épuration du lexique et une uniformisation de la langue. Comme le polar américain dont l'expression s'est construite à rebours des normes de l'anglais britannique (Tadié, 27), le Krimi se veut écrit dans une langue "populaire ${ }^{77}$. Cependant, il ne s'agit aucunement d'une langue oralisée (comme c'est le cas dans les fictions hard-boiled où le slang pénètre jusqu'à la voix narrative), mais bien d'un allemand standard compréhensible pour le plus grand nombre. On choisit parfois de germaniser les termes anglais ${ }^{78}$, mais surtout on évite le Rotwelsch, l'équivalent de l'argot, trop empreint de yiddish pour être toléré. Les différents personnages (bourgeois, policiers ou truands) parlent à quelques détails près la même langue et les accents $-\mathrm{y}$ compris dans la représentation de la sphère anglophone-sont effacés : dans 
Mord in Pittsburg (199), pour que l'enquêteur new-yorkais découvre qu'un suspect est australien, il faut qu'un troisième personnage l'en informe, aucun accent ne pouvant le trahir. En outre, on privilégie les phrases courtes, sans effet rhétorique appuyé, et les narrations simples: le récit se fait souvent en "focalisation zéro" et le discours indirect libre se raréfie. Il s'agit de retirer tous les éléments qui pourraient embrouiller le lecteur et nuancer ses certitudes. Cette tendance se fait sentir jusque dans les traductions. Les versions allemandes de certains crime novels réduisent les perspectives narratives (les discours directs et indirects libres deviennent des discours indirects, relevant d'un unique narrateur), explicitent les phrases ambiguës ou, au contraire, éludent les adjectifs et les adverbes qui connotent une incertitude (Sturge, 148-200). Plus encore, les passages décrivant les réflexions, les hypothèses et les tâtonnements de l'enquêteur sont effacés. Sous le Troisième Reich, le genre policier doit abandonner ses doutes, venus de l'étranger, et conforter la Weltanschauung germanique.

Il ne fait aucun doute que le genre policier a subi de profondes transformations sous le régime nazi. Cette acclimatation l'amène à se dépouiller de ses attributs traditionnels : aussi bien rationnels, démocratiques que judiciaires. Le style, les formes narratives, les personnages et les motifs explorés répondent à des discours idéologiques précis. Ces déformations se font à rebours du modèle anglais, convergent sans se confondre avec la production hard-boiled et laissent finalement apparaître un Krimi propre au nationalsocialisme. Cependant, si l'influence idéologique du nazisme sur le genre est avérée, il faut encore examiner la spécificité littéraire de la production : qu'est-ce qui distingue le Krimi d'une œuvre de propagande ou d'un document historique?

\section{L'exil imaginaire}

Le roman policier semble au service du régime parce qu'il met en avant un policier omnipotent, piétinant les lois pour anéantir les délinquants qui sont, eux, relégués dans l'ombre. Par cette "illégalité dans le droit» (voir Stolleis) et la mise au ban des criminels, le rapport centre-périphérie s'est inversé dans le Krimi. Comment peut toutefois perdurer le roman policier alors que la justice ne respecte plus les lois et que le crime est exclu de la nouvelle Allemagne?

La réponse la plus évidente consiste en un déplacement du Krimi en dehors de la sphère de références nazie. Bien entendu, ce phénomène ne concerne pas tous les romans. Toutefois, la plupart des œuvres se déroulent soit à l'étranger (en majorité en Angleterre ou aux États-Unis), soit dans l'Allemagne des siècles précédents (ce qui permet une résurgence de la tradition des Räuberromane, les romans de bandits aux accents schillériens) ou du moins avant 1933 (comme c'est le cas de Zehn einwandfreie Alibis qui se situe à Vienne en 1930). À moins qu'il ne s'agisse d'une Allemagne à l'époque imprécise : quelques éléments (téléphone, voiture, cinéma, etc.) évoquent les années 1920-1930, mais aucune référence n'est faite au Parti, à ses organisations parallèles et au mode de vie nazi. Personne ne fait le salut hitlérien et le culte de la personnalité s'est effacé. Dans l'ensemble, le Krimi ne prend pas place dans le Troisième Reich.

Cette étrangeté s'explique dans la logique du genre policier et de la littérature de divertissement. La forte présence de l'aire anglo-américaine peut être considérée comme un tribut payé aux initiateurs du genre. L'horizon d'attente du roman policier rend pour ainsi dire naturel l'enracinement de la fiction dans le sol anglais ou 
américain-et ce, parfois même en dépit de l'intrigue policière : dans Mord in Pittsburg, les meurtres sont commis soit en Australie, soit en Pennsylvanie, soit dans la campagne américaine, et pourtant l'enquête se déroule presque entièrement dans la ville de New York, topos du roman policier. À cela s'ajoute un exotisme facile, conséquence du repli nationaliste de l'Allemagne : les étrangers ont déserté le pays, Berlin a perdu le statut de ville cosmopolite qu'elle avait sous Weimar et, avec le tarissement des produits culturels importés, toute ouverture vers un ailleurs même occidental prend des parfums d'évasion. "Ce qui joue ici, c'est l'aventure romantique que représentent des villes cosmopolites de la taille de Londres et de New-York qui sont visités par des peuples étrangers, colorés et de tous les continents $»^{79}(1939,334)$, écrit Finke. Il faudrait ici évoquer brièvement la fonction des pays étrangers dans la topologie imaginaire du Troisième Reich : contrairement à l'Allemagne, où s'ancre la vérité, ils représentent des espaces de fantaisie d'où ne peuvent sortir que mensonges et histoires extravagantes. Ces histoires, c'est la propagande ennemie, ce sont les Gräuelmärchen ( « contes cruels ») colportés par la « juiverie mondiale » (Weltjudentum ; Klemperer, 57), mais aussi les romans policiers qui ne peuvent qu'être affabulations puisqu'étrangers : « D'emblée, nous savons qu'un brin de réalité est mélangé à tout un fagot d'inventions : et c'est justement ce qui nous attire $»^{80}$ (Franke-Heilbronn, 4).

Ce déplacement du Krimi dans un espace fantasmagorique peut lui être profitable, voire franchement libérateur. De fait, il permet d'imaginer un crime d'envergure qui, au regard des discours officiels, est désormais impossible en Allemagne. Il s'agit de placer les intrigues sous des latitudes exotiques où elles pourront s'épanouir. Dans Die Opiumhöhle von Cayenne (Cayenne, l'Enfer de l'opium), un truand résume bien la situation : "C'est que ce ne sont plus les mêmes institutions maintenant: des fonctionnaires incorruptibles! Avant, on réglait ça en vingt-quatre heures. Qu'est-ce que c'est compliqué aujourd'hui ! $»^{81}$ (Brock, 30) et décide de se tourner vers la Guyane. Le transfert de l'imaginaire criminel est ici d'autant plus ironique qu'il s'agit d'un gangster américain qui regrette le temps d'avant la "War on Crime ", c'est-à-dire avant 1933. Le déplacement du crime est effectivement l'occasion de jeux de miroir. Ainsi, Hauptzentrale New-York (Quartier général New York, Carstens, 1940) met en scène un réseau mafieux qui s'étend dans tous les États-Unis. «Hydra " possède une structure militaire, organisée en sections avec une police interne chargée d'exécuter les traitres et un chef suprême auxquels les bandits obéissent aveuglément : autant d'éléments qui peuvent rappeler le Parti nazi et qui avait valu au Testament du Dr. Mabuse (1933)de F. Lang d'être interdit à sa sortie. Mais il est vrai que le film se déroulait en Allemagne.

L'exil du Krimi lui alloue une certaine flexibilité dans son interprétation. Le roman peut se défendre de toute critique envers le régime et même prétendre s'accorder aux discours en vigueur. Le roman de Georg von der Vring, Spur im Hafen, qui se déroule dans l'Allemagne du XIX ${ }^{e}$ siècle, fait ainsi référence à la Révolution de Mars (1848) et ne cache pas sa sympathie envers les défenseurs de la liberté, appelant à la révolte contre toute oppression. Cependant, la critique de 1936 voit dans ces combattants des «hommes à l'esprit germanique » (deutschgesinnte Männer) qui combattent pour une "grande Allemagne » et rejoignent ainsi les idéaux du Reich (Adam, 192). Par ailleurs, d'autres auteurs ne manquent pas de souligner que si le Krimi se déroule avant tout à l'étranger, c'est parce que le pays a été purifié politiquement et spirituellement. Pour Finke, l'émigration du roman policier « découle de l'aversion des Allemands à laisser commettre un crime capital au sein de notre peuple, même si cela n'est que pure invention et littérature $»^{82}(1939,334)$. Ce déplacement corrobore l'idée que le criminel 
est toujours étranger à l'Allemagne, pays entouré de peuplades délinquantes. Il confirme aussi la supériorité du Reich sur les autres régimes occidentaux. Un critique se réjouit même que «par manque de [...] "liberté démocratique" » ${ }^{83}$, il ne puisse exister en Allemagne une « sous-humanité aussi répandue et aussi organisée qu'aux États-Unis en particulier $»^{84}$ (Diehl, 24).

Cette flexibilité a toutefois des limites et, à mesure que le régime se durcit, on exige du Krimi qu'il s'inscrive dans la nouvelle Allemagne. Erich Berger, dans un article de 1937 au titre éloquent (Marxismus im Romanteil ; "Marxisme dans le feuilleton-roman ») déplore que, malgré tous les efforts de propagande, les personnages n'appartiennent pas à l'aire culturelle nazie et conservent le « le caractère complet des sous-hommes ensauvagés $»^{85}(380)$. Langenbucher, quant à lui, ne décolère pas contre ces auteurs qui oublient leur devoir patriotique en plaçant leurs intrigues sous des latitudes étrangères : "On sent ici clairement que l'on veut se soustraire à la tâche que doit remplir un roman criminel qui se joue en Allemagne, entre Allemands $»^{86}$ (38). En effet, pour les membres les plus zélés de la RSK, le roman policier vaut surtout dans la lutte contre le crime et serait bien mieux employé dans le cadre du Troisième Reich : «La lutte contre les criminels et les asociaux doit être menée partout, y compris chez nous en Allemagne. Pourquoi ne pas décrire alors le combat de la police allemande contre la criminalité et la pègre ? $»^{87}$, exhorte Brunner-Ebersperg (14). Bien que les romans se déroulant à l'étranger constituent la majorité du corpus, on peut citer quelques Krimis qui rapatrient le genre en Allemagne nazie. C. Würmann (2004, 168-69) cite le cas de plusieurs enquêteurs qui, travaillant à l'étranger au début de l'intrigue, se sentent appelés par leur Nation et décident finalement de revenir sur le sol germanique. Ce Heimkehr ins Reich ( retour dans la patrie du Reich») constitue un motif récurrent du Krimi toutes tendances confondues et se fait plus sensible avec l'entrée en guerre de l'Allemagne. D'autres romans choisissent plus clairement de se placer sous l'égide de la police du Reich. C'est évidemment le cas de Der Tod fuhr im Zug: selon Alt dans sa préface (5), placer l'intrigue dans une ville fictive aurait détaché le crime de son lien nécessaire avec la communauté et affaibli la portée pédagogique du roman. Der Tod fuhr im Zug se déroule ainsi dans un Berlin explicitement nazi, où la guerre en cours et les institutions du régime sont clairement évoquées. La fonction de propagande est ici revendiquée, et ce, au détriment de l'aspect littéraire: Alt explique avoir renoncé à toute forme de "romançages » (Romantisierung) ou autre représentations qui auraient $\mathrm{pu}$ " assouplir » l'histoire racontée (7). Le Krimi, quand il est embrigadé par le Troisième Reich, perd l'attrait fantaisiste dont parlait Franke-Heilbronn et se détourne de l'Unterhaltungsliteratur.

Le Krimi venu de l'étranger peut représenter pour le lecteur un certain délassement idéologique-la Lockerung dont se défendait Alt-dans un régime de plus en plus rigide. Un libraire berlinois se souvient ainsi que les romans traduits étaient particulièrement prisés : « pour le public, c'était une garantie que ces œuvres n'avaient rien à voir avec la littérature prônée par le régime. Seule la littérature permettait une liaison spirituelle avec l'étranger $»^{88}$ (Benecke, 143). Le terme de geistiger Anschluss (« liaison, connexion, correspondance spirituelle ») est ici particulièrement pertinent car il pointe ce que le pouvoir nazi tentait justement d'étouffer : une circulation culturelle. Dans la LTI, le mot "Anschluss ", notamment dans le domaine littéraire, n'était compris que dans le sens d'une annexion et d'une mise au pas de l'étranger. Cet impérialisme culturel facilement observable dans le cas de l'Autriche-a pu souffrir dans un genre romanesque décidément allogène. Les Krimis dérangent l'ordre établi moins par leur 
exil imaginaire que par la communication qu'ils instaurent entre l'Allemagne et l'extérieur. Ils prennent appui sur les discours en vigueur tout en les relativisant ; ils font un « pli » sur des textes que l'on voudrait parfaitement lisses.

Cette circulation peut prendre la forme de références plus ou moins explicites aux discours nazis mais dans un cadre étranger. On a déjà évoqué ces effets de miroir. Certains romans peuvent faire preuve d'audace sous couvert d'une intrigue en Angleterre ou aux États-Unis, et ce, jusqu'à une époque tardive. Die Todeskabine ( $L a$ Cabine de la mort)de C.V. Rock, parue en 1939, met un détective aux prises avec une bande de criminels anglais. Leur chef, souvent comparé à un tyran, est contesté par ses hommes qui bientôt le destituent. Arrêté par le détective, le truand commente ainsi sa déchéance: "On pourrait peut-être la qualifier de "dictateur détrôné" ou de "fin précoce de la soif de pouvoir d'un despote". Mes hommes ne croyaient plus à l'ère glorieuse vers laquelle je voulais les mener et, comme mes instructions comportaient trop de risques, ils ont étouffé dans l'œuf mes velléités autocratiques $»^{89}(111)$. L'allusion est pour le moins troublante, d'autant plus qu'elle s'accompagne de critiques sur l'indiscipline des bandits, la désunion de la communauté criminelle et l'incapacité d'un chef à gouverner s'il n'a pas la même expérience vécue que son peuple: autant de motifs qui appartiennent au discours nazi et qui contribuent à l'ambivalence du roman.

Au-delà des références plus ou moins voilées, une forme littéraire en particulier permet une circulation discursive plus frondeuse: les aveux épistolaires-dont on trouve quelques exemples dans la nouvelle Eine Gewissensbeichte (Une Confession)de M. Zwick et Hauptzentrale NewYork de B. Carstens. Ces lettres fictives que rédigent les criminels pour se confesser aux policiers constituent un geistiger Anschluss remarquable dans le contexte du Troisième Reich, puisqu'elles font communiquer une parole extérieure avec le discours nazi tout en inversant les rapports de pouvoir. Elles s'inscrivent d'une part dans une tradition d'écriture populaire : les lettres que, depuis le XIX siècle et plus encore après 1933, les nombreux émigrés allemands envoient d'Amérique à leurs compatriotes, leur racontant leurs difficultés d'intégration mais aussi les libertés acquises dans l'exil ${ }^{90}$. D'autre part, comme nous l'avons dit, le régime a particulièrement valorisé l'aveu dans sa pratique judiciaire. Les procédures d'enquête et d'interrogatoire ont été remaniées pour obtenir la confession du coupable (Wagner, 1996, 193-213). La détention préventive et les pratiques musclées ont permis de faire avouer des criminels célèbres comme Franz et Erich Sass en 1940-ce que la police ne se prive pas de claironner. Cette recherche de l'aveu participe d'une évolution plus large des logiques de pouvoir et de la criminologie. Foucault (1976, 76-94) a souligné que, au moins depuis le XVIe siècle, les sociétés occidentales étaient devenues particulièrement " avouantes» (dans la justice, la religion, l'amour, la médecine, etc.). À la fin du XIX siècle, des criminologues comme Lombroso ou Lacassagne incitent les détenus à raconter leur histoire pour en tirer le portrait d'une personnalité criminelle. L'aveu est le signe d'une victoire de la société disciplinaire. Cette victoire est double dans le cas de la confession épistolaire du Troisième Reich puisque si le criminel envoie une lettre à la police, c'est parce qu'il a dû fuir l'Allemagne, décidément trop bien surveillée. Or, les injonctions à avouer et l'exil forcé du crime engendrent des textes particulièrement ambigus. Une confession épistolaire signifie la fuite du criminel devant la puissante Kripo mais aussi son impunité. Un personnage écrit au commissaire car il lui est désormais possible « d'échapper à l'acuité de votre œil policier-si puissant par ailleurs $»^{91}$. À la fois reconnaissance et transgression du pouvoir policier, les lettres 
aiment à rappeler cet éloignement salvateur dans les contrées imaginaires du crime-le plus souvent aux États-Unis. Dans Hauptzentrale New York, Fred Iverster, le chef de l'organisation "Hydra ", a rédigé un cahier qu'il devait envoyer à la police après sa fuite. Le truand a déjà été tué lorsqu'on découvre ses feuillets et, d'outre-tombe, il continue de narguer les agents (338-346). De même, la lettre d'Eine Gewissenbeichte de Zwick débute ainsi : "Cher Monsieur le Préfet de police,/ Maintenant que je suis en parfaite sécurité, [...], je puis me permettre de vous parler franchement car... je vous suis désormais inaccessible $»^{92}$. L'éloignement ouvre un espace de liberté car ce que l'on ne se risquait pas à dire peut désormais s'écrire. La sécurité (Sicherheit) géographique, c'est-à-dire être hors de portée de la police (Sicherheitsamt), permet de parler aufrichtig, c'est-à-dire sincèrement mais aussi honnêtement, conformément à ce qui est juste (richtig; Recht). Les savoureuses formules de politesse évoquent le droit (dürfen, erlauben)que se donne le scripteur face à une police hors-la-loi. La forme épistolaire en elle-même est un motif de trouble car elle implique une absence à la première personne. Alors que, dans la culture de masse, le criminel est éclipsé, il réapparaît ici certes en exil mais au premier plan de la narration. La lecture se fait au plus près du « je » criminel, tandis que le destinataire fictif-le policier-est tenu au silence. Par les actes ou la parole, il ne peut répliquer à cette lettre car le coupable est parti sans laisser d'adresse: comme écrit malicieusement le narrateur d'Eine Gewissenbeichte, il est unerreichbar («injoignable» mais aussi «hors de portée»). Le pacte épistolaire renverse ici les relations de pouvoir entre la police et les marginaux du Troisième Reich.

Pourtant, la communication s'établit, notamment parce que la confession épistolaire s'inscrit dans le discours criminologique nazi. Au-delà de la pratique de l'aveu, les personnages sont bel et bien allemands car ils font écho aux conceptions en vigueur-à savoir l'image d'un criminel doté d'une volonté mauvaise ou trop faible pour résister aux tentations, à moins qu'il ne soit racialement dégénéré. Dans ces écrits de coupables en exil, les thèses volontaristes et biologiques sont bien présentes mais subverties, comme si l'éloignement permettait leur déformation. L'assassin d'Eine Gewissensbeichte se définit lui-même comme un homme doté d'une grande volonté qui agit avec un «sang-froid instinctif» (instinktiv kaltblütig; 119) et qui est motivé par la vengeance familiale, une vengeance qui coule littéralement dans ses veines et « qui s'apparente à la vengeance de sang de certaines peuplades $\|^{93}$ (120). Plus clairement encore, le truand scripteur de Hauptzentrale NewYork se révèle être le jumeau du commissaire, battant en brèche les études qui, durant les années 1930, ont affirmé que la gémellité garantissait un «destin criminel» aux deux individus ${ }^{94}$. Dans sa confession, le criminel précise même qu'il avait « hérité » (vererbt; mot appartenant au lexique biologique ; 339) des mêmes traits de caractère que son frère mais qu'il a basculé dans le crime après avoir raté sa carrière d'artiste. Ce détail ne peut que troubler le lecteur allemand de 1940, abreuvé de la biographie du Führer. Sans faire d'Hauptzentrale NewYork un brûlot de résistance, il introduit une correspondance subversive entre l'Allemagne nazie et l'imaginaire étranger.

49 Le rapport du Krimi à l'étranger, le plus souvent sur le mode du refus et de l'absence, façonne la production du Troisième Reich au moins à trois égards. Concernant les affiliations littéraires, le roman policier britannique fournit l'horizon d'attente, les modèles et contre-modèles du genre, tandis que la production américaine propose plutôt un cadre imaginaire où le crime peut se déployer. Même quand les œuvres étrangères disparaissent du marché allemand, elles définissent en creux le Krimi. 
Deuxièmement, la xénophobie culturelle du Troisième Reich fait émerger une littérature criminelle spécifique à ce régime. Des auteurs allemands profitent de l'espace vacant et, par delà l'habituelle dichotomie du roman policier, le Krimi se construit selon une troisième voie, à rebours de la tradition anglaise et à l'écart du polar américain. On pourrait presque parler d'un Krimi proprement national-socialiste -aussi bien dans les motifs que dans la forme-qui renverse les configurations du polar américain: le détective hard-boiled ne commet plus ses transgressions en marge du système légal, il disparaît au profit du policier allemand qui occupe le centre du Krimi et place les écarts de conduite au cœur de la justice nazie. Cette confiscation du pouvoir policier et l'introduction d'un arbitraire, subordonné à la Weltanschauung, tend à faire du Krimi non pasun roman de la société disciplinaire, mais bien une fiction totalitaire.

Cependant, le genre policier allemand se révèle indocile du fait même de son rapport à l'étranger. Les mesures coercitives nazies ont transformé les États-Unis en un espace de liberté, de fantaisie et de subversion-des latitudes qui attirent le Krimi vers un exil fictif. Suite aux pressions du régime, l'échange culturel et le dialogue littéraire se transforment en la persistance d'un ailleurs dans l'imaginaire du Troisième Reich : une échappatoire, mais aussi une remise en cause des normes en vigueur.

Pourtant, il faut reconnaître que cette nationalisation forcée du Krimi a aussi permis une première différenciation de la production allemande face aux romans britanniques et américains. Que cet héritage soit assumé ou non, la période nazie contient les germes du polar qui va éclore après 1945. Ainsi, Horst Bosetzky en narrant l'histoire du tueur en série Paul Orgozow dans Wie ein Tier-Der S-Bahn-Mörder (1995) reprend une célèbre histoire criminelle de 1940-1941 et, surtout, s'appuie sur le roman d'Axel Alt, Der Tod fuhr im Zug, qui inaugurait en 1944 une collection policière "dans l'air du temps » (neuzeitliche Kriminalromane). Ce moment oublié de l'histoire littéraire a posé les jalons du polar moderne en Allemagne-avec toutes les tensions qui constituent l'identité du roman germanique d'après-guerre.

\section{BIBLIOGRAPHIE}

ALT, Axel, Der Tod fuhr im Zug. Den Akten der Kriminalpolizei nacherzählt, Berlin-Leipzig, Verlag Hermann Hillger, 1944.

ANONYME, « Arbeitstagung der Reichschrifttumsstelle für die Verleger von Unterhaltungs - und Volksliteratur ", Börsenblatt für den Deutschen Buchhandel, vol. CII, n² 276, 28 novembre 1935, 1015.

ANONYME, « Möglichkeiten des Kriminalromans », Die Literatur Monatschrift für Literatursfreunde, vol. XLI, $\mathrm{n}^{\circ} 10$, juillet 1939, 583 .

ADAM, Christian, Lesen unter Hitler. Autoren, Bestseller, Leser im Dritten Reich, Berlin, Galiani, 2010.

ARENDT, Hannah, Le Système totalitaire, traduit de l'anglais par Jean-Loup Bourget, Robert Davreu et Patrick Lévy, Paris, Éditions du Seuil, 1972. 
BARBIAN, Jan-Pieter, Literaturpolitik im NS-Staat. Von der « Gleichschaltung » bis zum Ruin, Francfort/ Main, Fischer Taschenbuch Verlag, 2010.

BENECKE, Hans, Eine Buchhandlung in Berlin : Erinnerungen an eine schwere Zeit, Francfort/Main, Fischer Taschenbuch Verlag, 1995.

BERGER, Erich, « Marxismus im Romanteil », Deutsche Presse Zeitschrift des Reichsverbandes der deutschen Presse, vol. XXVII, $\mathrm{n}^{\circ}$ 24, 21 août 1937, 380-81.

BEST, Werner, Die deutsche Polizei. Darmstadt, L. C. Wittich, 1940.

BROCK, Rudolf, Die Opiumhöhle von Cayenne, Berlin, Eden-Verlag, 1936.

BRUNNER-EBERSPERG, G., « Wie stehen wir zum Kriminalroman? Kriminalroman : Ja oder nein ? », Die Zeitschrift der Leihbücherei, vol. 11, n 1, 10 janvier 1942, 14-15.

CARSTENS, Bernd (pseud. de Josef HERRMANN), Hauptzentrale New York, Berlin, Kulturelle Verlagsgesellschaft, 1940.

CZECH, Hans-Jörg et Nkola DOLL (dir.), Kunst und Propaganda im Streit der Nationen 1930-1945, Berlin - Dresde, Deutsches historisches Museum - Sandstein Verlag, 2007.

DALY, Carroll J., The Snarl of the Beast, Boston, Gregg Press, 1981 (@1927).

---, « Three Gun Kelly », in W. Nolan (dir.), The Black Mask Boys, (1921.

DELEUZE, Gilles, « Philosophie de la Série Noire », L'île déserte et autres textes, Les éditions de Minuit, Paris, 2002, 114-24.

DIEHL, Edgar « Eine Lanze für den Kriminalroman », Großdeutsches Leihbüchereiblatt, vol. I, n 1, 1939, 23-24.

DOMARUS, Max, Hitler : Reden und Proklamationen 1932-1945 Kommentiert von einem Zeitgenossen, vol. I : Triumph (1932-1938), Neustadt a. d. Aisch, Verlagsdruck Schmidt, 1962.

DYKE, James van, « Über die Beziehungen zwischen Kunst, Propaganda und Kitsch in Deutschland 1933 bis 1945 », dans Hans-Jörg Czech et Nkola Doll (dir.), Kunst und Propaganda im Streit der Nationen 1930-1945, Berlin - Dresde, Deutsches historisches Museum - Sandstein Verlag, 2007, 250-57.

EICHBERG, Arnold, « Der Kriminalromanleser. Eine psychologische Studie. », Die Zeitschrift der Leihbücherei, vol. VI, nº 18-19, 25 septembre 1937 - 10 octobre 1937, 10-11.

Ero, « Über den Kriminalroman », Bücherkunde, vol. vI, n³, mars 1939, 154-55.

FINKE, Edmund, « Über den Kriminalroman », Die Literatur. Monatschrift für Literatursfreunde, vol. VI, $n^{\circ} 41$, mars 1939, 333-35.

---,. Zehn einwandfreie Alibis. Berlin-Vienne-Leipzig, Karl H. Bischoff Verlag, 1942.

FOUCAULT, Michel, Surveiller et Punir. Naissance de la prison, Paris, Gallimard, 1975.

---, Histoire de la sexualité, tome I, La Volonté de savoir, Paris, Gallimard, 1976.

FRAENKEL, Ernst, The Dual State: A Contribution to the Theory of Dictatorship, traduit de l'allemand par E. A. Shils, New York, Oxford University Press, 1941.

FRANKE-HEILBRONN, Hans, « Der Kriminal-,Schmöker' als Moritaten-Ersatz. Kleine Betrachtung über ein großes Feld der Literatur », die Zeitschrift der Leihbücherei, vol. IX, nº 13, 10 juillet 1940, $4-5$. 
GASSERT, Philipp, Amerika im Dritten Reich. Ideologie, Propaganda und Volksmeinung 1933-1945, Stuttgart, Steiner, 1997.

GEYER-RYAN, Helga, « Wunschkontrolle - Kontrollwünsche. Die Gleichschaltung der Populärliteratur im Dritten Reich », dansJörg Thunecke (dir.), Leid der Worte. Panorama des literarischen Nationalsozialismus, Bonn, Bouvier Verlag, 1987, 177-206.

GILMAN, Sander L. (dir.), NS-Literaturtheorie Eine Dokumentation, Francfort s./Main, Athenäum Verlag, 1971.

HASPER, Eberhard, « Zustand und Zukunft der deutschen Volksliteratur », Börsenblatt für den Deutschen Buchhandel, vol. CII, 170, 25 septembre 1935, 608.

HAYCRAFT, Howard, Murder for Pleasure. The Life and Times of the Detective Story, Londres, Peter Davies, 1941.

HOYER, Walter, « Was schadet der Kitsch?», Börsenblatt für den Deutschen Buchhandel, vol. cIV, $\mathrm{n}^{\circ}$ 77, 6 avril 1937, 303.

HELBICH, Wolfgang (dir.), "Amerika ist ein freies Land ..." : Auswanderer schreiben nach Deutschland, Darmstadt, Luchterhand, 1985.

KRANZ, Heinrich, Lebensschicksale krimineller Zwillinge, Berlin, Springer, 1936.

KLEMPERER, Viktor, LTI, la langue du troisième Reich, traduit de l'allemand par Elizabeth Guillot, Paris, Albin Michel, 1996, 202 [Leipzig, Reclam Verlag, 1975].

LANGE, Johannes, Verbrechen als Schicksal : Studien an kriminellen Zwillingen, Leipzig, Thieme, 1929.

LANGENBUCHER, Erich, « “Der Teufel spielt Verstecken” oder Einiges zur Frage des gegenwärtigen Kriminalromans », Die Buchbesprechung : Eine monatliche Umschau, vol. III, $\mathrm{n}^{\circ} 2$, février 1939, 37-41.

LINDER, Joachim, « Feinde im Inneren. Mehrfachtäter in deutschen Kriminalromanen der Jahre 1943/44 und der "Mythos Serienkiller" ", Internationales Archiv für Sozialgeschichte der deutschen Literatur, vol. XXVIII, $\mathrm{n}^{\circ} 2,2003,190-227$.

LOSCH, Sebastian, « Unterhaltungsschriftum der Leihbüchereien - so oder so ? », Großdeutsches Leihbüchereiblatt, vol. II, $\mathrm{n}^{\circ}$ 4, avril 1940, 41-42.

MARCUS, Steven, « Introduction », Dashiell Hammett,The Continental Op, New York, Vintage Books, 1992 (@1974), vii-xxiv.

MAGNY, Claude-Edmonde, L’Âge du roman américain, Paris, Éditions du Seuil, 1948.

MAYER-EBELING, Alf, « Der Mann, der uns Chandler und Hammett brachte. Krimipionier Karl Anders und seine Krähen-Bücher », dans Nina Schindler dir., Das Mordsbuch. Alles über Krimis. Hildesheim, Claassen, 1997,102-09.

MORIN, Edgar, L'Esprit du temps, essai sur la culture de masse, t. I : Névrose, Paris, Grasset, 1962. NOLAN, William B. (dir.), The Black Mask Boys : Masters in the Hard-Boiled School of Detective, New York, William Morrow and Company, 1985.

NUSSER, Peter, Der Kriminalroman, Stuttgart - Weimar, Metzler, 1992.

REICHSSCHRIFTTUMSKAMMER, Schriftsteller-Verzeichnis, Leipzig, Reichsschrifttumskammer, 1942. 
RIX, Walter T., « Wesen und Wandel des Detektivromans im totalitären Staat », dans Paul G. Buchloh et Jens P. Becker dir., Der Detektivroman. Studien zur Geschichte und Form der englischen und amerikanischen Detektivliteratur. Darmstadt, Wissenschaftliche Buchgesellschaft, 1978, 121-134.

ROCK, C.V. (Kurt Walter Roecken), Gangster in der Prärie, Berlin : Kulturelle-Verlag-Gesellschaft, 1937.

---, Die Todeskabine, Berlin , A. Weichert Verlag, 1939.

RÜTHERS, Bernd, Die unbegrenzte Auslegung zum Wandel der Privatrechtsordnung im Nationalsozialismus, Tübingen, Mohr, 1968.

PLATINI, Vincent, « Éclipse, exil et survie du Krimi en Allemagne nazie (1933-1945) », Belphégor, Littérature populaire et culture médiatique, «Polar et politique », vol. IX, n³, décembre 2010, en ligne, http://etc.dal.ca/belphegor/vol9_no3/articles/09_03_vincen_survie_fr.html (page consultée le 01/12/2011).

SAUPE, Achim, Der Historiker als Detektiv - der Detektiv als Historiker : Historik, Kriminalistik und der Nationalsozialismus als Kriminalromann, Bielefeld, Transcript-Verlag, 2009.

SCHÄDEL, Mirko, Illustrierte Bibliographie der Kriminalliteratur 1796-1945 im deutschen Sprachraum. Butjadingen, Achilla Presse Verlagsbuchhandlung, 2006.

SCHIVELBUSCH, Wolfgang, Entfernte Verwandtschaft : Faschismus, Nationalsozialismus, New Deal 1933 - 1939, Munich, Carl Hanser Verlag, 2005.

SPIEKER, Markus, Hollywood unterm Hakenkreuz : Der amerikanische Spielfilm im Dritten Reich, Trier, WVT Wissenschaftlicher Verlag, 1999.

STIEG, Margaret, Public Libraries in Nazi Germany, Tuscalosa, University of Alabama Press, 1992.

STOLLEIS, Michael, Recht im Unrecht : Studien zur Rechtsgeschichte des Nationalsozialismus. Francfort/ Main, Suhrkamp, 1994.

STUMPFL, Friedrich, Ursprünge des Verbrechens : Dargestellt am Lebenslauf von Zwillingen, Leipzig, Thieme,1936.

STURGE, Kate, «The Alien Within » : Translation into German during the Nazi Regime, Munich, Iudicium Verlag, 2004.

TADIÉ, Benoît, Le Polar américain : la modernité et le mal, Paris, PUF, 2006.

THIELKE, Karl, « Der englische Kriminalroman », Die Buchbesprechung, vol. III, n 3, 1939, 42-43.

TOBOS, A. (pseudonyme de Kurt SOBOTA), Der Mord in Pittsburg, Dresde, Seyfert, 1939.

TODOROV, Tzvetan, « Typologie du roman policier », Poétique de la prose, Paris, Éditions du Seuil, 1971, 55-65.

VRING, Georg von der, Die Spur im Hafen. Roman, Güterloh, Scherl, 1936.

WAGNER, Patrick, Volksgemeinschaft ohne Verbrecher : Konzeptionen und Praxis der Kriminalpolizei in der Zeit der Weimarer Republik und des Nationalsozialismus, Hambourg, Christian, 1996.

---, Hitlers Kriminalisten. Die deutsche Kriminalpolizei und der Nationalsozialismus zwischen 1920 und 1960, Munich, C.H. Beck Verlag, 2002.

WEIGAND, Jörg, « C.V. Rock : Lust am präzisen Ausdruck », Das weite Feld der Phantasie : Aspekte deutschsprachiger Unterhaltungsliteratur ; 111 Aufsätze aus 28 Jahren, Passau, Erster Dt. Fatasy-Club e.V., 1996, 189-90. 
WERNERT, Schickert, « Absinken des Kriminalromans? », Die Literatur, vol. v, n³5, février 1933,

247.

WOLF, Nelly, Le Roman et la démocratie, Saint-Denis, Presses Universitaires de Vincennes, 2003.

WÜRMANN, Carsten, « Zum Kriminalroman im National-Sozialismus » dans Carsten Würmann et Bruno Franceschini, dir., Verbrechen als Passion. Neue Untersuchungen zum Kriminalgenre, Berlin, Weidler Buchverlag, 2004, 143-86.

---, Zwischen Unterhaltung und Propaganda. Das Krimigenre im Dritten Reich, thèse de doctorat soutenue à la Freie Universität de Berlin, Berlin, 2008.

ZWICK, Michael, 8 Minuten und andere Kriminalfälle. Berlin, Eden-Verlag, 1934.

\section{NOTES}

1. Parmi les plus célèbres, citons: August Gottlieb Meißner (1753-1807) dans la tradition des crimes et procès célèbres; Friedrich Schiller avec Der Verbrecher aus verlorener Ehre (1786) ou encore E.T.A. Hoffmann avec Das Fräulein von Scuderi (1819).

2. À la suite notamment de l'article pionnier de Walter Rix (1973) sur la production des fictions policières dans les régimes totalitaires.

3. Sur l'état des recherches concernant le Krimi allemand et une évaluation plus complète de la production sous le Troisième Reich, je renvoie à mon article paru en 2010 dans la revue Belphegor et consultable en ligne.

4. Le Börsenblatt für den Deutschen Buchhandel est l'organe du Börsenvereins der Deutschen Buchhändler, fédération nationale des éditeurs et diffuseurs allemands.

5. «Eine nationalsozialistiche Schrifttumpsflege handelt positiv. Das einzige Verfahren, das auf die Dauer Erfolg verspricht, heißt, das wertlose Buch durch das werthafte zu überwinden und verdrängen. »

6. DAF ; organisation inféodée au régime, fondée le 10 mai 1933 et qui se substitue aux syndicats sous le Troisième Reich.

7. Ainsi que le note déjà Edgar Morin, la culture de masse, justement parce qu'elle est commerciale, fait preuve d'une certaine "élasticité ", produit d'un dialogue entre une production et une consommation (Morin, 56).

8. Dirigée par Alfred Rosenberg, nommé en 1934 « Beauftragten des Führers für die Überwachung der gesamten geistigen und weltanschaulichen Schulung und Erziehung der NSDAP » ( Mandataire du Führer pour la surveillance de l'ensemble de la formation et de l'éducation spirituelles et idéologiques du NSDAP »), cet office de surveillance publiait de nombreuses recensions et avis d'interdiction-en concurrence avec le ministère du Reich à l'Éducation du peuple et à la Propagande (Reichsministerium für Volksaufklärung und Propaganda, RMVP). Son organe est le très influent mensuel Die Bücherkunde, qui a paru entre 1934 et 1944.

9. Schriftsteller-Verzeichnis 1942.

10. Il faut cependant se garder d'une dichotomie entre des auteurs amateurs forcément émancipés et des écrivains professionnels inféodés au régime: la plupart des amateurs cherchaient à intégrer la RSK pour des raisons de prestige et de confort financier (Barbian, 396 sqq.) tandis qu'un auteur aux écrits pour le moins séditieux comme Adam Kuckhoff figurait encore sur les listes de la RSK en 1941, soit un an avant l'arrestation qui devait mener à son exécution pour sa participation active depuis 1933 à un groupe de résistance.

11. «Was er [der Staat] nicht kann, ist: selbst Volksliteratur schreiben. Darin liegt die Hauptschwerigkeit. » 
12. C'est par ailleurs en ce sens que Michel Foucault considère le roman policier depuis le XIX ${ }^{\mathrm{e}}$ siècle : un corollaire de l'avènement de la société disciplinaire $(1975,82)$.

13. Notre propos rejoint ici les réflexions de Nelly Wolf (2003) qui voit dans le pacte de lecture romanesque une reproduction imaginaire du contrat démocratique. Par ailleurs, ce débat sur l'aspect démocratique du roman policier émerge dès 1940 dans l'étude classique de Howard Haycraft (311): «the detective story is and always has been essentially a democratic institution; produced on any large scale only in democracies; dramatizing under the bright cloak of entertainment, many of the precious rights and privileges that have set the dwellers in constitutional lands apart from those less fortunate ».

14. «Das Lesepublikum der ganzen Welt hatte sich bis in die letzten Jahre damit abgefunden, daß England auf dem Gebiet des Kriminalromans sozusagen ein "Monopol" besaß. »

15. Grünes Eis, trad. de Hansi Bochow Blüthgen, Leipzig, E. Oldenburg Verlag 1931.

16. Auge um Auge (Murder Won't Wait), trad. de Fritz Pütsch, Leipzig: Goldmann Verlag, 1934; Die verborgene Hand (The Hidden Hand) trad. de Hansi Bochow-Blüthgen, Leipzig: Ernst Oldenburg Verlag, 1933; Der weisse Diamant (The Amateur Murderer), trad. de Fritz von Bothmer, Leipzig: Goldmann Verlag, 1934 - réed. en 1939.

17. Ein Blick zurück (The Case of the Sulky Girl), trad. par Anton Zahorsky, Vienne: Alfred Ibach Verlag; Rechtanswalt Mason (The Case of the Velvet Claws) trad. par Hans Herdegen, Berlin: Kulturelle Verlagsgesellschaft, 1933;Die sechs Glasaugen (The Case of the Counterfeit Eye),trad. par Kurt Ziegler, Vienne: Alfred Ibach Verlag, 1937.

18. En 1933, on compte 41 films américains sur 81 films étrangers à l'écran contre 129 allemands ; en 1936, cependant, on ne compte plus que 28 films américains sur 64 films étrangers contre 112 allemands.

19. « in der Richtung der leuchtenden Stadt, deren hohe Bauten wie in einem Trickfilm am Horizont emporwuchsen... »

20. «Poste de consultation pour la littérature de divertissement", cabinet créé en 1935 et dépendant de la RSK.

21. Paraissant entre 1935 et 1943 et appartenant à la sphère d'influence de Rosenberg et des SS, la revue mensuelle Weltliteratur: Romane, Erzählungen und Gedichte aller Zeiten und Völker ( Littérature du monde : romans, récits et poèmes de tout temps et de tous les peuples ») a une vocation explicitement internationale, défendant une conception de la traduction en tant qu'acte éminemment politique contre la « juiverie anglo-américaine » qui domine la littérature mondiale (Sturge, 104-115).

22. "Wir lehnen alle Autoren, die gegen uns hetzen, $a b$ ».

23. «Der englische Kriminalroman ist für den deutschen immer vorbildlich gewesen ».

24. « ein Film, der ein Beispiel dafür sein kann, wie man Geschehnisse knapp, logisch, sachlich gestalten muß ».

25. Au demeurant, comme le souligne Gassert (180-181), le Troisième Reich n'entretient pas une relation de franche antipathie avec les États-Unis du New Deal qui sont plutôt perçus - du moins jusqu'en 1937-1938 - comme un modèle à suivre et à dépasser dans le domaine de la rationalisation du travail et de l'industrie cinématographique.

26. «Man weiß, daß viele ausländische Kriminalromane einen großen Erfolg hatten und zugegeben - teilweise als Vorbild für nicht untüchtige Schriftsteller gedient haben, deshalb nützt man die unverständliche Anhänglichkeit aus... »

27. «Es ist an der Zeit, daß diejenigen, die Kriminalschriftsteller sein wollen, sich gründlich wandeln. Denn lange werden sie keine Früchte mehr solcher Saat ernten ».

28. " Es wird nicht schwer sein, die Dutzendware eines Edgar Wallace aus dem Feld zu schlagen und dafür etwas zu schaffen, was der stille Wunsch vieler Kriminalromanleser seit langem ist, den guten deutschen Kriminalroman ». 
29. " eine deutliche Verherrlichung englischer Einrichtungen und englischer Lebensart darstellt oder die Arbeit der englischen Polizei in den Vordergrund stellt».

30. «Sie muß von feindstaatlichen Einflüssen völlig frei sein und sich, was das Gebiet des Kriminalromans anbetrifft, jeder Bezugsnahme auf Scotland Yard und die englische Polizei enthalten ".

31. «Ein deutscher Sherlock Holmes wäre auch mal ganz nett! »

32. «Diese Gestalt ist eine so spezifisch englische Erfindung, daß jegliche Nachahmung ausgeschlossen ist » (ibid., $154:$ « ce personnage est une invention si spécifiquement anglaise que toute imitation est exclue »).

33. «Der Kriminalroman behandelt, moralisch betrachtet, im Grunde nichts anderes als [...] den Krieg der Ordnungsmächte gegen das zuchtlose Chaos der Unterwelt. »

34. «Verdient die deutsche Polizei nicht der wirkliche Held solcher Bücher zu sein?»

35. «Einzelheiten [...], die zwar vom Standpunkt des Kriminalisten und auch hinsichtlich des Motivs von Bedeutung, aber für uns im Rahmen der Darstellung unwichtig sind. »

36. Sur ce transfert de valeurs du serial-killer au policier du Reich, $c f$. Linder.

37. Cette éclipse explique en partie la place relativement faible que tiennent les personnages de criminels juifs dans les Krimis du Troisième Reich, contrastant avec l'acharnement de la propagande officielle. Ce type de truand est d'autant mis à l'écart que les juifs étaient exclus de la sphère publique et de son imaginaire. En fait, seules les publications les plus proches du Parti (comme les feuilletons publiés par le Völkischer Beobachter) mettent systématiquement et explicitement en scène cette figure criminelle.

38. LaReichsschrifttumsstelle communique, le 28 novembre 1935, après ses journées de travail sur la littérature populaire la résolution suivante: «Es müsse seitens der Vertreter des deutschen Rechts die Forderungerhoben werden, daß der Kriminalroman sowie der Kriminalfilm in Zukunft nicht mehr von reißerischen Spannungsmomenten getragen werden dürfen " («Les représentants de la loi allemande doivent exiger que le roman et le film criminels ne comportent plus, à l'avenir, de sensationnels moments de suspens », cit. in Geyer-Ryan 1987, 185).

39. «Sind Passfälschungen, Devisenvergehen, Kindsraub, Autoräubereien keine ernsten Probleme?».

40. « das Verbrechen [...] nichts weiter als Imbezilität und falsch orientierte Trägheit erweist, da der Verbrecher regelmäßig mit den schwierigsten Mitteln die geringsten Erfolge erzielt und ein Risiko trägt, das in gar keinem Verhältnis zum eventuellen „Nutzen“ der Tat steht. »

41. Le parallèle est d'autant plus tentant que Kurt Müller, sous le pseudonyme de Hermann Hilgendorff, met en scène un G-Man dans une série de romans intitulée "Jack Kelly " et qui compte 28 fascicules en 1937. Malheureusement, nous n'avons pu retrouver ces aventures du FBI en version allemande dans les fonds de bibliothèque que nous avons consultés.

42. Le prologue du film The Beast of the City(La Bête de la cité, Charles Brabin, 1932) signé par Hoover, l'annonce clairement: "instead the glorification of the gangster we need the glorification of the policemen...».

43. Respectivement 'G'Men (Hors-la-loi, 1935) et Bullets or Ballots (Guerre au crime, 1936) de W. Keighley.

44. Notons néanmoins que la convergence de l'Amérique du New Deal et de l'Allemagne nazie a déjà appelé à quelques comparaisons chez les historiens. Voir: Schivelbusch; Czech et Doll.

45. «Der Polizei als Hüterin der Ordnung steht in den zahlreichsten Fällen der soviel klügere Privatdetektiv gegenüber ».

46. «Es geht nicht an, daß der Privatdetektiv Held der Handlung bleibe ».

47. On peut à ce propos citer la conception du droit qu'expose Adolf Hitler dans sa Regierungserklärung («déclaration de gouvernement») devant le Reichstag le 23 mars 1933 : «Unser Rechtswesen muß in erster Linie der Erhaltung dieser Volksgemeinschaft dienen. [...] Nicht das Individuum kann Mittelpunkt der gesetzlichen Sorge sein, sondern das Volk.» 
(« Notre droit doit en premier lieu servir la conversation de la communauté raciale. [...] Ce n'est pas l'individu qui doit être au centre des considérations légales, mais bien le peuple»; cit. in Domarus, 233).

48. « Die deutsche Polizei ist für uns ja keine Behörde oder kein Instrument der Staatsmacht an sich allein, sondern auf ihrem Gebiet die Repräsentation der deutschen Volksgemeinschaft. »

49. Rock, 184: « Hm, dann wollen wir also wieder einmal von der Regel abweichen! »

50. Cette distinction se fait à l'encontre de l'imaginaire du genre policier lui-même qui, depuis les années 1970, a souvent placé ses récits hard-boiled dans le décor du Troisième Reich (Saupe 302-08).

51. Ainsi que le résume Terry Mack, un des premiers détectives hard-boiled créé par Daly:«I ain't a crook, and I ain't a dick; I play the game on the level, in my own way. I'm in the center of a triangle; between the crook and the police and the victim » $(1923,43)$.

52. «Seine Gangstergewohnheiten hatte Bobby allerdings noch nicht aufgegeben. »

53. " Erst wollte der Wirt nicht mit der Sprache heraus, aber - na du kennst ja meine Leute schließlich ließ er sich überreden. »

54. « Es war verdammt ungesetzlich! »; « Was Durop getan hat, war noch viel ungesetzlicher! »

55. La Verordnung des Reichspräsidenten zum Schutze von Volk und Staat (« décret du Président du Reich sur la protection du peuple et de l'État ») fait suite à l'incendie du Reichstag et apparaît pour certains (Fraenkel) comme le texte constitutionnel du régime nazi. Il supprime notamment les articles de l'ancienne Constitution garantissant la liberté individuelle, d'expression et de presse tout en limitant fortement le droit à la propriété privée.

56. « Damit müssen - ohne förmliche Aufhebung oder Abänderung der einzelnen älteren Gesetze - alle abweichenden rechtlichen Bestimmungen als im Sinne der neuen Auffassung abgeändert gelten. »

57. « Eine gesunde Verwaltung hat mit Paragraphen überhaupt nichts zu tun.»

58. «Ich bin sonst rücksichtsvoll in meinem Vorgehen! Aber jetzt ist Schluß damit! Von nun an wird geschossen!»

59. C'est ce que Bernd Rüthers a analysé sous le nom d' «interprétation illimitée » (unbegrenzte Auslegung). Il cite notamment Carl Schmitt qui écrit en 1934: "Das gesamte heutige deutsche Recht, einschließlich der weitergeltenden, positiv nicht aufgehobenen Bestimmungen muß ausschließlich und allein vom Geist des Nationalsozialismus beherrscht sein. [...] Jede Auslegung muß eine Auslegung im nationalsozialistischen Sinne sein» («Nationalsozialismus und Rechtsstaat ", Juristische Wochenschrift, 1934, cit. in Rüthers, 175 : « L'ensemble du droit allemand actuel, y compris les anciennes dispositions toujours valides, dont la valeur positive n'a pas été abrogée, doit exclusivement être régi par l'esprit du national-socialisme. [...] Toute interprétation doit être interprétation dans un sens national-socialiste »).

60. Encore en 1941, un critique fustige les romans policiers anglo-saxons dans lesquels «schweigt das Schicksal und der Tod ist tot " (Günther Helmut, "Der Kriminalroman und die angelsächsischen Länder », cit. in Würmann, 2004, 156 : « le destin se tait et la mort est morte »).

61. «Wo geistreiche Gentlemanverbrecher geschildert werden, will der Kriminalschriftsteller die Gefahren eines verantwortungslosen Intellektualismus darstellen, der die Welt entgöttert und zu einem Tummelplatz von kalt genossen liebelosen Leidenschaften macht. »

62. Pour une présentation plus complète de cette dichotomie critique, voir Tadié, 8-13.

63. «einem warmfühlenden Herzen und echter, durch Erfahrung erworbener Menschenkenntnis. »

64. « [er] fühlt instinktiv, daß der Kerl die Wahrheit sprach » (je souligne).

65. «Chappell liebte solche Schauspiele. Er war der Meinung, daß so eine dramatische Mitteilung außerordentlich aufschlussreich sein konnte. Und die Erfahrung gab ihm darin Recht. »

66. « Nur ich kann die Augen ein bißchen weiter aufmachen! »

67. À ce sujet, voir Marcus (1992, 7-24) et Tadié (88-91). 
68. «wir fingieren nicht einmal gern eine Welt in der das Verbrechen sozusagen hinter jeder nächsten Tür geschehen kann. »

69. « Du bist ein mißtrauriger Halunke [...] Als ich bei ihm eingestellt wurde, machte er [mein Chef]mir zur Bedingung, immer das zu sagen, was ich gegen meinen Gegner zu unternehmen gedächte.»

70. «Wenn Sie [...] nicht die große Scheu vor der Öffentlichkeit gehabt hätten, so würde es nie einen Mordfall Sprigg gegeben. »

71. Ainsi, on peut lire dans Die Literatur (Anonyme, juillet 1939, 583): «Vermutlich liegt es an einer Eigentümlichkeit unserer Sprache; wir könnten uns denken, dass schon in ihr, in ihren ursprünglichen Gehalten und Wortausstrahlungen ein Widerstand gegen das seelisch eingeengte, künstliche Wesen des Kriminalromans ruht » («cela tient probablement à une propriété de notre langue. On peut concevoir en elle, dans ses idées originelles, dans la puissance évocatoire de ses mots, une résistance à l'artificialité et à l'étroitesse d'âme du roman policier »).

72. «Was allein durch derartige Machwerke an Sprach-Verziehung verschuldet wird, ist kaum auszudrücken », déplore Langenbucher $(37$; « on peine à dire ce qui est à mettre sur le seul compte de ces torchons pour ce qui est de la difformité de la langue »).

73. « unsere Muttersprache wird in viele dieser Erzeugnisse auf jeder Seite vergewaltigt. »

74. « Deutsch sein, heißt klar sein.»

75. «Das Einfache in der Dichtung "; « Alles Schöne ». Ce discours a été tenu le 10 octobre 1942 lors des très officielles rencontres des écrivains à Weimar (Dichtertreffen) organisées dans le cadre de la «semaine du livre allemand». Le discours de Vring a été partiellement reproduit dans le Börsenblatt du 17 octobre 1942. Vring conclut notamment son discours par un exhortation à remplacer le kitsch par la simplicité dans la littérature populaire.

76. « sprachlich und schriftstellerisch anständig ».

77. C'est du moins ce qu'affirment Georg von der Vring (1942) et Langenbucher (40). Sur l'aspect populaire de la LTI, on consultera également Klemperer (299-302).

78. Dans une certaine mesure, le Krimi évite les mots étrangers, quand il ne procède pas à une véritable domestication linguistique : à titre d'exemple, dans Mord in Pittsburg, le quartier newyorkais de Chinatown est germanisé en «Chinesenstadt ». On se gardera cependant de grossir le trait et l'on renverra à l'étude de Sturge (171) qui relève des exemples de Krimis (y compris ceux écrits par Edmund Finke) qui ne craignaient pas les anglicismes.

79. « [S]pielt dabei die abenteuerliche Romantik riesiger Weltstädte von den Ausmaßen Londons und New York mit, die von fremden, auch farbigen Völkern aller kontinente besucht warden. »

80. "Wir wissen von vornherein, daß hier ein Gran Wirklichkeit mit einem Bündel von Erfindunden gemischt ist: und gerade das ist es, was uns hinreißt. »

81. «Das ist auch so eine neue Einrichtung, unbestechliche Beamte! Früher war so etwas in vierundzwanzig Stunden geregelt. Was für Komplikationen heute! »

82. «entspringt der Abneigung der Deutschen, Kapitalverbrechen in den Grenzen des eigenen Volkstums spielen zu lassen, auch dann, wenn sie bloß erfunden und erdichtet sind. »

83. « mangels [...]"demokratischer Freiheit" »

84. «so ausgedehntes und wohlorganisiertes Untermenschentum wie ganz besonders in den Vereinigten Staaten »

85. «Charakter völlig verwilderter Untermenschen. »

86. «Man spürt dabei sehr deutlich, daß man sich damit vor der Verantwortung drücken will, die ein in Deutschland selbst, unter deutschen Menschen spielender Kriminalroman zu erfüllen hat. "

87. «Der Kampf gegen verbrecherische Menschen und asoziale Elemente muß überall geführt werden, auch bei uns in Deutschland. Warum also nicht den Kampf der deutschen Polizei gegen Verbrechertum und Unterwelt beschreiben. " 
88. «für das Publikum war das eine Garantie dafür, daß diese Werke nichts mit der offiziell gewünschten Literatur zu tun hatten. Geistiger Anschluss an das Ausland wurde nur durch Literatur möglich [...]. »

89. «Das könnte man vielleicht bezeichnen mit "enthronter Diktator" oder "frühzeitiges Ende der Machtgelüste eine Herrschsüchtigen". Meine Leute glaubten nicht mehr an die herrlichen Zeiten, denen ich sie entgegenführen wollte, und haben, da mit meinen Anordnungen ein größeres Risiko verbunden war, meine autokratischen Bemühungen im Keime erstickt. »

90. Pour trouver quelques exemples de ces lettres, voir Helbich, 110-127.

91. « dem Bannkreis Ihrer sonst so scharfen Augen zu entfliehen ».

92. « Herr Polizeipräsident! Heute, wo ich mich in völliger Sicherheit befinde, [...] darf ich es mir erlauben, aufrichtig mit Ihnen zu reden, denn... ich bin für Sie unerreichbar. »

93. « die der Blutrache verschiedener Völker verwandt ist. »

94. Parmi les criminologues qui ont défendu cette thèse, citons Johannes Lange (1929), Friedrich Stumpfl (1936) et Heinrich Kranz (1936).

\section{RÉSUMÉS}

L'article considère le genre policier sous le Troisième Reich et sa relation à la production angloaméricaine dans une perspective économique, idéologique et formelle. Le Krimi s'inscrit dans une logique de marché lui permettant de faire valoir des nécessités commerciales à côté des impératifs politiques. Cette logique lui aménage un espace réduit de liberté et le place en concurrence avec la production étrangère qui domine largement le marché. À mesure que le régime affirme son totalitarisme, les œuvres traduites se raréfient et les pressions idéologiques se font sentir sur le Krimi : le genre policier prend des formes dictatoriales, issues des pratiques judiciaires de la nouvelle Allemagne, et se forme à rebours du roman de tradition anglaise. Bien que la convergence entre Krimi nazi et polar hard-boiled soit esquissée, la production allemande se distingue clairement de l'imaginaire des pays démocratiques. Cependant et en dépit des pressions idéologiques, le Krimi n'est pas complètement assujetti au national-socialisme : il garde des teintes étrangères et, par des exils imaginaires, se permet de narguer le pouvoir nazi.

This article deals with the specificity of the crime novel under the Third Reich and its relation to the Anglo-American genre, from an economic, ideological and formal perspective. As a marketable commodity, the so-called Krimi was informed by commercial as well as political imperatives. This logic granted it a small degree of freedom and placed it in competition with the foreign products that dominated the literary market. However, with the increasing totalitarianism of the regime, translated novels became scarcer and ideological pressures exerted themselves heavily on the Krimi. The crime novel then took on dictatorial forms, reflecting the changed conception of the law in the new Germany and contrasting with the English tradition of the genre. Although Nazi Krimis and American hard-boiled stories converged to a certain extent, the German products differed unmistakably in their opposition to the imagination prevalent in democratic countries. Nevertheless, despite ideological pressures, the Krimi never became entirely subservient to the regime : it continued to harbor foreign influences and, by launching into imaginary exiles, even managed to mock the power of Nazism. 
INDEX

Keywords : crime novel, economic aspect, Germany, ideology, image studies, imaginary exile, resistance, Third Reich

Mots-clés : Allemagne, aspect économique, exil imaginaire, idéologie, imagologie, résistance, roman policier, Troisième Reich

\section{AUTEUR}

\section{VINCENT PLATINI}

Université Paris 4 (CRLC) / Freie Universität Berlin 